\title{
The Quantum Zero Space Charge Model for Semiconductors *
}

\author{
Andreas Unterreiter ${ }^{\dagger}$ \\ Fachbereich Mathematik \\ Universität Kaiserslautern \\ D - 67653 Kaiserslautern, Germany
}

\begin{abstract}
The thermal equilibrium state of a bipolar, isothermal quantum fluid confined to a bounded domain $\Omega \subset \mathbb{R}^{d}, d=1,2$ or $d=3$ is the minimizer of the total energy $\mathcal{E}_{\epsilon \lambda} ; \mathcal{E}_{\epsilon \lambda}$ involves the squares of the scaled Planck's constant $\epsilon$ and the scaled minimal Debye length $\lambda$. In applications one frequently has $\lambda^{2} \ll 1$. In these cases the zero-space-charge approximation is rigorously justified. As $\lambda \rightarrow 0$, the particle densities converge to the minimizer of a limiting quantum zero-space-charge functional exactly in those cases where the doping profile satisfies some compatibility conditions. Under natural additional assumptions on the internal energies one gets an differential-algebraic system for the limiting $(\lambda=0)$ particle densities, namely the quantum zero-space-charge model. The analysis of the subsequent limit $\epsilon \rightarrow 0$ exhibits the importance of quantum gaps. The semiclassical zero-space-charge model is, for small $\epsilon$, a reasonable approximation of the quantum model if and only if the quantum gap vanishes. The simultaneous limit $\epsilon=\lambda \rightarrow 0$ is analyzed.
\end{abstract}

\section{Introduction}

Quantum hydrodynamic models (QHDs) give a fairly accurate account of the macroscopic behavior of ultra-small semiconductor devices in terms of only macroscopic quantities such as particle densities, current densities and electric fields [5].

The thermal equilibrium state of QHDs is the minimizer of the energy functional [9]

$$
\mathcal{E}_{\epsilon \lambda}(n, p) \equiv \epsilon^{2} \mathcal{E}_{q u}(n, p)+\mathcal{E}_{i}(n, p)+\lambda^{-2} \mathcal{E}_{e l}(n, p)
$$

in

$$
\mathcal{C} \equiv\left\{(n, p) \in L^{1}(\Omega) \times L^{1}(\Omega): n, p \geq 0, \sqrt{n}, \sqrt{p} \in H^{1}(\Omega), \int_{\Omega} n=N, \int_{\Omega} p=P\right\},
$$

where all involved quantities are dimensionless, $\epsilon$ is the scaled Planck's constant, $\lambda$ is the scaled minimal Debye length and

$$
\mathcal{E}_{q u}(n, p) \equiv \int_{\Omega}|\nabla \sqrt{n}|^{2}+\int_{\Omega}|\nabla \sqrt{p}|^{2}
$$

\footnotetext{
${ }^{*}$ To appear in the European Journal of Applied Mathematics.

†The author acknowledges support from EC-network, contract \# ERBCHRXCT 930413, from the project entitled "Mathematische Analysis und Numerik von Quantenhydrodynamischen Modellen in der Halbleiterphysik" funded by the Deutsche Forschungsgemeinschaft and from the grant ERBFMRXCT970157 (TMR-Network) from the EU.
} 
is the scaled quantum energy,

$$
\mathcal{E}_{i}(n, p) \equiv \int_{\Omega} G_{1}(n)+\int_{\Omega} G_{2}(p)
$$

is the internal energy and

$$
\mathcal{E}_{e l}(n, p)=\frac{1}{2} \int_{\Omega}|\nabla \Phi[n-p-C]|^{2}
$$

is the scaled electrostatic energy.

The charge carriers of the semiconductor device are considered as quantum fluids. The non-void, bounded domain $\Omega \subseteq \mathbb{R}^{d}, d=1,2$ or $d=3$, represents the semiconductor device. The spatial variable $x$ ranges through $\Omega$. The functions $n=n(x)$ and $p=p(x)$ are particle densities of negatively-charged electrons and positively-charged holes. It is assumed that the total number $N$ of electrons and $P$ of holes is prescribed. The internal energy $\mathcal{E}_{i}$ takes into account many-particle effects. The strictly convex functions $G_{1,2}$ are primitives of the enthalpy functions $g_{1,2}$ of the electron- and hole-gas, respectively. $g_{1,2}$ are related to the respective pressure functions ${ }^{1} r_{1,2}:\left[0, \infty\left[\rightarrow\left[0, \infty\left[\operatorname{via} g_{1,2}(\rho)=\right.\right.\right.\right.$ $\int_{1}^{\rho}\left(r_{1,2}^{\prime}(t)\right) / t d t$. The doping profile $C \in L^{\infty}(\Omega)$ represents a fixed background charge density. It is assumed that $N=\rho_{i}+\int_{\Omega} C^{+}$and $P=\rho_{i}+\int_{\Omega} C^{-}$where $\rho_{i}>0$ is an intrinsic constant. The scaled electrostatic potential $\Phi$ equals $\Phi[n-p-C]$ where for $f \in L^{2}(\Omega)$ with $\int_{\Omega} f=0$ the function $\Phi[f]$ is given as unique $H^{1}(\Omega)$-solution of Poisson's equation

$$
-\Delta \Phi[f]=f \quad, \quad \int_{\Omega} \Phi=0
$$

i.e. we assume that $\int_{\Omega} \Phi=0$ and the validity of the variational identity ${ }^{2}$

$$
\forall \phi \in H^{1}(\Omega): \quad \int_{\Omega} \nabla \Phi[f] \cdot \nabla \phi=\int_{\Omega} f \phi .
$$

The energy functional $\mathcal{E}_{\epsilon \lambda}$ involves two dimensionless parameters, namely $\epsilon, \lambda$. In applications one frequently has [2]

$$
\lambda^{2} \ll 1
$$

One may expect that the smallness of $\lambda^{2}$ expresses some redundancy of the model equations. The question arises whether the thermal equilibrium solution of the QHD is close to the solution (if it exists at all) of a limiting model where $\lambda$ is set to zero. Such models will be called "quantum zero-space-charge models" ("QZSCs"). Typical for QZSCs is the involvement of quantum mechanical terms and the replacement of Poisson's equation by the "zero-space-charge assumption" $n-p-C=0$. Whether QZSCs are a good approximation or not depends not only on the order of magnitude of $\lambda^{2}$ but also on the ration of $\epsilon^{2}$ and $\lambda^{2}$ which equals

$$
\rho \equiv \frac{\epsilon^{2}}{\lambda^{2}}=K \hbar^{2} \frac{\sup _{\Omega}\left|C^{*}\right|}{\left(T^{*}\right)^{2}}
$$

where $K$ is a constant, $C^{*}$ is the unscaled doping profile and $T^{*}$ is an unscaled reference temperature of the charge carrier gases. Due to the orders of magnitude of $C^{*}$ and $T^{*}$ one has to distinguish between three cases:

\footnotetext{
${ }^{1}$ Pressure functions are typically strictly increasing and continuously differentiable on $[0, \infty)$.

${ }^{2}$ It is easy to see that $\Phi[f]$ satisfies homogeneous Neumann-boundary conditions.
} 
1.) $\rho \ll 1$, which corresponds to high temperature and moderate doping. As $\epsilon^{2} \ll$ $\lambda^{2} \ll 1$ the model equations are "almost semi-classical" and one is compelled to analyze the consecutive limits $\epsilon \rightarrow 0, \lambda \rightarrow 0$.

2.) At low temperature and moderate or high doping one has $\rho \gg 1$ and the limit $\lambda^{2} \rightarrow 0$ has to be investigated. Depending on the order of magnitude of $\epsilon^{2}$ quantum effects may play a major role if $\epsilon^{2}$ is not negligible or are of minor importance if $\epsilon^{2} \ll 1$. If $\epsilon^{2} \ll 1$ holds then the subsequent limit $\epsilon \rightarrow 0$ will be of interest.

3.) If $\rho \approx 1$ then $\epsilon^{2} \approx \lambda^{2} \ll 1$ and we will be concerned with the simultaneous limit $\lambda=O(\epsilon) \rightarrow 0$.

The limits of 1.) have been analyzed in [9].

The aim of the present paper is to perform a rigorous analysis of the remaining limits. For the first time, we will derive a quantum-zero-space charge model.

The several steps of the analysis are as follows. For $\epsilon, \lambda \in] 0, \infty[$ we denote the (unique) minimizer of $\mathcal{E}_{\epsilon \lambda}$ in $\mathcal{C}$ by $\left(n_{\epsilon \lambda}, p_{\epsilon \lambda}\right)$. The corresponding ground energy is $E_{\epsilon \lambda} \equiv$ $\mathcal{E}_{\epsilon \lambda}\left(n_{\epsilon \lambda}, p_{\epsilon \lambda}\right)=\inf _{\mathcal{C}} \mathcal{E}_{\epsilon \lambda}(n, p)$. Theorem 1 is concerned with the case where $\epsilon$ is fixed and $\lambda$ tends to 0 . One gets $n_{\epsilon \lambda}-p_{\epsilon \lambda}-C \rightarrow 0$ in an apropriate sense, i.e. the zero-spacecharge approximation is asymptotically correct for $\lambda \rightarrow 0$. Theorem 1 deals with the value $E_{\epsilon} \equiv \lim _{\lambda \rightarrow 0} E_{\epsilon \lambda} \in[0, \infty]$. A reasonable QZSC can only arise in cases where $E_{\epsilon}$ is finite, because otherwise the system's ground energy tends to infinity when $\lambda \rightarrow 0$. It turns out that $E_{\epsilon}<\infty$ is equivalent to the assumption that the doping profile $C$ is a $\left(G_{1}, G_{2}\right)$-quantum doping profile. If $C$ has an abrupt junction, then $C$ will not be a $\left(G_{1}, G_{2}\right)$-quantum doping profile. Hence it is not reasonable to employ abrupt junctions in quantum models which include the zero-space-charge approximation.

Theorem 3 is concerned with a closer investigation of $\left(n_{\epsilon \lambda}, p_{\epsilon \lambda}\right)$ as $\lambda \rightarrow 0$. $\left(n_{\epsilon \lambda}, p_{\epsilon \lambda}\right)$ tends to the minimizer $\left(n_{\epsilon \circ}, p_{\epsilon \circ}\right)$ of a quantum zero-space-charge functional. The corresponding Euler-Lagrange equations constitute the QZSC. It follows from Theorem 4 that $\left(n_{\epsilon \circ}, p_{\epsilon \circ}\right)$ is a solution of this QZSC.

Concerning the consecutive limit $\epsilon \rightarrow 0$, a consistency problem arises. It cannot be excluded that the ground energy of the limiting semi-classical model is not the limit of quantum ground energies. Whenever such a "quantum-gap" appears, then the limiting problem is inconsistent with the quantum picture and the semi-classical model gives no justifiable approximation. Theorem 5 is concerned with the case where no quantum-gap appears. Then the limit $\epsilon \rightarrow 0$ leads to the semi-classical zero-space-charge drift-diffusion equations and the limits 1.) and 2.) commute.

Theorem 6 deals with the simultaneous limit $\lambda=\epsilon \rightarrow 0$.

The proofs of the results are deferred to the appendix. 


\section{The Quantum Zero-Space-Charge Model}

The subsequent investigations are based on the following assumptions:

(A)

$$
\begin{aligned}
& \text { a) } \Omega \subset \mathbb{R}^{d}, d=1,2 \text { or } d=3 \text { is a bounded domain with } \partial \Omega \in C^{0,1} . \\
& \text { b) There exists a } K>0 \text { such that for all } f \in L^{2}(\Omega) \text { with } \int_{\Omega} f=0 \\
& \qquad\|\Phi[f]\|_{L^{\infty}} \leq K\|f\|_{L^{2}} . \\
& \text { c) } C \in L^{\infty}(\Omega) . \\
& \text { d) } \left.N-P=\int_{\Omega} C, N=\rho_{i}+\int_{\Omega} C^{+}, P=\rho_{i}+\int_{\Omega} C^{-}, \rho_{i} \in\right] 0, \infty[. \\
& \text { e) } g_{1,2} \in C(] 0, \infty[) \cap L_{l o c}^{1}([0, \infty[) \text { is strictly increasing, } \\
& \lim _{t \rightarrow \infty} g_{1,2}(t)=\infty \text { and } \underline{g_{1,2}} \equiv \lim _{t \rightarrow 0+} g_{1,2}(t) \in[-\infty, \infty[.
\end{aligned}
$$

In accordance with e) we can choose $s_{1,2} \in[0, \infty[$ such that for all $s \in[0, \infty[$

$$
G_{1,2}(s) \equiv \int_{s_{1,2}}^{s} g_{1,2}(\sigma) d \sigma \geq 0 .
$$

As usual we identify $f \in L^{2}(\Omega)$ with the functional

$$
\begin{aligned}
f: H^{1}(\Omega) & \rightarrow \mathbb{R} \\
\phi & \mapsto \int_{\Omega} f \phi
\end{aligned}
$$

which obviously belongs to $\left(H^{1}(\Omega)\right)^{\prime}$.

The proof of the following result can be found in [9]:

Assume $(\mathbf{A})$. Then $\mathcal{E}_{\epsilon \lambda}$ has exactly one minimizer $\left(n_{\epsilon \lambda}, p_{\epsilon \lambda}\right)$ in $\mathcal{C}$. Furthermore,

a) $\left(n_{\epsilon \lambda}, p_{\epsilon \lambda}\right)$ is a weak solution of the Euler-Lagrange equations

$$
\left.\begin{array}{rl}
\epsilon^{2} \Delta \sqrt{n_{\epsilon \lambda}} & =\sqrt{n_{\epsilon \lambda}}\left(\lambda^{-2} \Phi_{\epsilon \lambda}+g_{1}\left(n_{\epsilon \lambda}\right)+\alpha_{\epsilon \lambda}\right) \\
\epsilon^{2} \Delta \sqrt{p_{\epsilon \lambda}} & =\sqrt{p_{\epsilon \lambda}}\left(-\lambda^{-2} \Phi_{\epsilon \lambda}+g_{2}\left(p_{\epsilon \lambda}\right)+\beta_{\epsilon \lambda}\right) \\
-\Delta \Phi_{\epsilon \lambda} & =n_{\epsilon \lambda}-p_{\epsilon \lambda}-C
\end{array}\right\}
$$

where $\alpha_{\epsilon \lambda}, \beta_{\epsilon \lambda} \in \mathbb{R}$ and $\int_{\Omega} n_{\epsilon \lambda}=N, \int_{\Omega} p_{\epsilon \lambda}=P, \int_{\Omega} \Phi_{\epsilon \lambda}=0$.

b) Each of the three equations of (1) can be tested with any $\phi \in H^{1}(\Omega)$.

c) $n_{\epsilon \lambda}, p_{\epsilon \lambda}, \Phi_{\epsilon \lambda} \in H^{1}(\Omega) \cap L^{\infty}(\Omega)$.

We define for $\epsilon, \lambda \in] 0, \infty\left[\right.$ the ground energy $E_{\epsilon \lambda} \equiv \inf _{\mathcal{C}} \mathcal{E}_{\epsilon \lambda}=\min _{\mathcal{C}} \mathcal{E}_{\epsilon \lambda}$ and for $\epsilon \in] 0, \infty\left[\right.$ let $E_{\epsilon} \equiv \lim _{\lambda \rightarrow 0} E_{\epsilon \lambda}$. Clearly $E_{\epsilon} \in[0, \infty]$ and for fixed $\left.\epsilon \in\right] 0, \infty$ [ the function $\lambda \mapsto E_{\epsilon \lambda}$ is decreasing. Hence $E_{\epsilon}=\sup _{\lambda \in] 0, \infty[} E_{\epsilon \lambda}$.

The asymptotic validity of the zero-space-charge approximation is the content of

Theorem 1 Assume (A). Let $\epsilon \in] 0, \infty[$. Then: 
a) $\Phi_{\epsilon \lambda} \rightarrow 0$ strongly in $H^{1}(\Omega)$ as $\lambda \rightarrow 0$.

b) $n_{\epsilon \lambda}-p_{\epsilon \lambda}-C \rightarrow 0$ strongly in $\left(H^{1}(\Omega)\right)^{\prime}$ as $\lambda \rightarrow 0$.

c) $\lim _{\lambda \rightarrow 0} \lambda^{2} E_{\epsilon \lambda}=0$.

The zero-space-charge approximation is asymptotically correct. But to achieve a reasonable limiting model the ground state energy has to be bounded as $\lambda \rightarrow 0$. This is however not always the case.

Theorem 2 Assume (A). Then i), ii), iii) are equivalent.

i) For all $\epsilon \in] 0, \infty\left[: E_{\epsilon}<\infty\right.$.

ii) There is an $\epsilon \in] 0, \infty\left[\right.$ with $E_{\epsilon}<\infty$.

iii) There exists $\left(n^{*}, p^{*}\right) \in \mathcal{C}$ such that $C=n^{*}-p^{*}$ and $\mathcal{E}_{i}\left(n^{*}, p^{*}\right)<\infty$.

An equivalent formulation of the condition on $C$ in ii) is

Lemma 1 Assume (A). Then i), ii) are equivalent.

i) $C=r^{2}-s^{2}$ with $r, s \in H^{1}(\Omega)$.

ii) $C=f_{1} f_{2}$ with $f_{1} \in H^{1}(\Omega), f_{1} \geq 0$ and $f_{2} \in H^{1}(\Omega) \cap L^{\infty}(\Omega)$.

Statement i) deserves some further comments. To require that $C$ is the difference of squares of $H^{1}(\Omega)$-functions is consistent with the quantum picture. The doping profile $C=C^{+}-C^{-}$represents a background distribution of charged particles. The densities $C^{ \pm}$of these particles are given by squares of moduli of Schrödinger functions $\Psi^{ \pm}$solving a stationary Schrödinger equation on $\Omega$. Hence $\Psi^{ \pm}$naturally belong to $H^{1}(\Omega)$ and because of $C=C^{+}-C^{-}=\left|\Psi^{+}\right|^{2}-\left|\Psi^{-}\right|^{2}$ the doping profile $C$ is actually the difference of squares of $H^{1}(\Omega)$-functions. This observation motivates the following definition.

Definition 1 Assume (A). Then $C$ is called a $\left(G_{1}, G_{2}\right)$-quantum doping profile, provided there exists $\left(n^{*}, p^{*}\right) \in \mathcal{C}$ such that

$$
C=n^{*}-p^{*} \quad \text { and } \quad \mathcal{E}_{i}\left(n^{*}, p^{*}\right)=\int_{\Omega} G_{1}\left(n^{*}\right)+\int_{\Omega} G_{2}\left(p^{*}\right)<\infty .
$$

With this definition we can re-formulate Theorem 2 as follows: For fixed $\epsilon \in] 0, \infty[$ the ground energy is bounded independently of $\lambda \in] 0, \infty\left[\right.$ if and only if $C$ is a $\left(G_{1}, G_{2}\right)$ quantum doping profile.

In the sequel most of the results will concern $\left(G_{1}, G_{2}\right)$-quantum doping profiles. Hence it is extremely important to have criteria which allow to decide whether $C$ is a $\left(G_{1}, G_{2}\right)$ quantum doping profile or $\operatorname{not}^{3}$. We recall that (A) implies that $C \in L^{\infty}(\Omega)$.

Lemma 2 Assume (A). Then:

1. If $d=1$, then $C$ is a $\left(G_{1}, G_{2}\right)$-quantum doping profile if and only if $C \in H^{1}(\Omega)$.

2. If $C \in H^{1}(\Omega)$, then $C$ is a $\left(G_{1}, G_{2}\right)$-quantum doping profile.

\footnotetext{
${ }^{3}$ The author is indebted to the anonymous referee for essential parts of the subsequent discussion.
} 
3. If $d=2$ and if $C$ is a $\left(G_{1}, G_{2}\right)$-quantum doping profile, then for all $q \in[1,2[$ : $C \in W^{1, q}(\Omega)$.

4. If $d=3$ and if $C$ is a $\left(G_{1}, G_{2}\right)$-quantum doping profile, then $C \in W^{1,3 / 2}(\Omega)$.

Maybe the most important consequence of Lemma 2 is the fact that piecewise constant (or piecewise smooth) doping profiles with abrupt junctions are not $\left(G_{1}, G_{2}\right)$-quantum doping profiles. This is surprising because we deduce from Theorem 2 (as well as from the subsequent discussion) that these widely used doping profiles do not allow for a physically acceptable quantum zero-space-charge model.

On the other hand, any doping profile in $H^{1}(\Omega)$ (e.g., each doping profile in $C^{1}(\bar{\Omega})$ ) is a $\left(G_{1}, G_{2}\right)$-quantum doping profile and a reasonable limiting $(\lambda \rightarrow 0)$ model is available.

In applications one usually has two types of models for $C$. On the one hand there are piecewise constant doping profiles with abrupt junctions. In the context of semiconductor device modeling these doping profiles are admittedly approximations of the "realistic" distribution of background charges. A close observation of the fabrication procedure shows that the available doping profiles look much more like uniformly continuous functions whose gradients are locally "large" but globally bounded, i.e. $C \in W^{1, \infty}(\Omega)$. Hence from an application's point of view, Lemma 2 gives a satisfactory answer to the question which doping profile is a $\left(G_{1}, G_{2}\right)$-quantum doping profile and which is not.

From a mathematical point of view, Lemma 2 does not cover the question whether each $\left(G_{1}, G_{2}\right)$-quantum doping profile belongs to $H^{1}(\Omega)$ or not. While this is true in one-dimensional settings, the case $d \geq 2$ is extremly complicated and far beyond the scope of this paper. However, to get an impression of the difficulties in connection with this question some aspects of the case $d=2$ are discussed in the "Conclusions".

On the other hand, we have the following result which shows that each $\left(G_{1}, G_{2}\right)$ quantum doping profile is "not far away from $H^{1}(\Omega)$ ". Let $S$ be the unit sphere $\{\xi \in$ $\left.\mathbb{R}^{d}:|\vec{\xi}|^{2}=1\right\}$ and for $\vec{\xi} \in S$ let $\vec{\xi}^{\perp}:=\left\{\vec{v} \in \mathbb{R}^{d}: \vec{v} \cdot \vec{\xi}=0\right\}$. For $\vec{\xi} \in S$ and $\vec{v} \in \vec{\xi}^{\perp}$ we introduce

$$
I(\vec{\xi}, \vec{v}):=\{t \in \mathbb{R}: \vec{v}+t \vec{\xi} \in \Omega\} .
$$

Then $I(\vec{\xi}, \vec{v})$ is an open subset of $\mathbb{R}$ and we have

Lemma 3 Assume (A), $d \geq 2$ and let $C$ be a $\left(G_{1}, G_{2}\right)$-quantum doping profile. Then for all $\vec{\xi} \in S: C$ has a representative $c$ (i.e. $c: \Omega \rightarrow \mathbb{R}$ is measurable and $C=\{d: \Omega \rightarrow$ $\mathbb{R}: c=d$ almost everywhere $\})$ such that for almost all $\vec{v} \in \vec{\xi}^{\perp}$ with $I:=I(\vec{\xi}, \vec{v}) \neq \emptyset$ :

$$
c(\vec{v}+t \vec{\xi}) \in H^{1}(I) \cap L^{\infty}(I)
$$

Let us consider

Example 1 We set $\Omega:=] 0,1[\times] 0,1[$.

a) It is easy to see that the function $C_{1}: \Omega \rightarrow \mathbb{R},(x, y) \rightarrow C_{1}(x, y):=-(1 / 2)+\sqrt{x}$ belongs to $W^{1, q}(\Omega) \cap L^{\infty}(\Omega)$ for all $q \in[1,2[$. Hence we can not decide by means of Lemma 2 whether $C_{1}$ is a $\left(G_{1}, G_{2}\right)$-quantum doping profile or not. However we have for all $y \in] 0,1[$ : The function $] 0,1\left[\ni x \rightarrow C_{1}([], y).(x):=C_{1}(x, y)=-(1 / 2)+\sqrt{x}\right.$ does not belong to $H^{1}(] 0,1[)$. Hence by Lemma 3: $C_{1}$ is not a $\left(G_{1}, G_{2}\right)$-quantum doping profile.

b) In similiarity to a) the reader may wish to verify that $C_{2}: \Omega \rightarrow \mathbb{R},(x, y) \rightarrow C_{2}(x, y):=$ $-(1 / 2)+\sqrt{x y}$ not a $\left(G_{1}, G_{2}\right)$-quantum doping profile.

c) Since $C_{3}: \Omega \rightarrow \mathbb{R},(x, y) \rightarrow-(1 / 2)+\sqrt{x^{2}+y^{2}}$ belongs to $H^{1}(\Omega) \cap L^{\infty}(\Omega)$ we have: 
$C_{3}$ is a $\left(G_{1}, G_{2}\right)$-quantum doping profile.

d) Now consider

$$
\begin{aligned}
& C_{4}: \quad \Omega \quad \rightarrow \quad \mathbb{R} \\
& (x, y) \mapsto\left\{\begin{array}{rl}
1 & , \quad 0 \leq x<1 / 2 \\
-1 \quad & 1 / 2 \leq x<1
\end{array} .\right.
\end{aligned}
$$

$C_{4}$ represents a doping profile with an abrupt junction. We already know that $C_{4}$ is not a $\left(G_{1}, G_{2}\right)$-quantum doping profile. One may however ask how to find apropriate "regularizers" $C_{4}^{\delta}$ of $C_{4}$ which are $\left(G_{1}, G_{2}\right)$-quantum doping profiles. A possibility would be

$$
\begin{aligned}
C_{4}^{\delta:} \quad \Omega & \rightarrow \mathbb{R} \\
(x, y) & \mapsto\left\{\begin{array}{rl}
1 & 0 \leq x<(1 / 2)-\delta \\
1-\frac{2(x-(1 / 2)+\delta)^{\alpha}}{(2 \delta)^{\alpha}}, & (1 / 2)-\delta \leq x<(1 / 2)+\delta \\
-1 \quad, & (1 / 2)+\delta \leq x<1
\end{array}\right.
\end{aligned}
$$

where $\delta \in] 0,(1 / 2)[$ and $\alpha \in] 0, \infty\left[\right.$. According to the discussion of a) we easily get: $C_{4}^{\delta}$ is $a\left(G_{1}, G_{2}\right)$-quantum doping profile if and only if $\alpha>1 / 2$.

Physically reasonable quantum zero-space-charge models can only arise for uniformly bounded ground energies which is the case iff $C$ is a $\left(G_{1}, G_{2}\right)$-quantum doping profile. But what happens if $C$ is not a $\left(G_{1}, G_{2}\right)$-quantum doping profile? A partial answer to this question is given in

Lemma 4 Assume (A). Let $\epsilon \in] 0, \infty\left[\right.$. Let $q \in\left[1, \infty\left[\right.\right.$ such that $L^{2 q}(\Omega)$ is continuously embedded in $H^{1}(\Omega)$. Assume there is $\left.\epsilon \in\right] 0, \infty[$ such that

or

i) $\lim \sup _{t \rightarrow \infty} G_{1,2}(t) / t^{q}<\infty \underline{\text { and }} \lim \sup _{\lambda \rightarrow 0} \mathcal{E}_{q u}\left(n_{\epsilon \lambda}, p_{\epsilon \lambda}\right)<\infty$

ii) $\lim \sup _{\lambda \rightarrow 0}\left\|\lambda^{-2} \Phi_{\epsilon \lambda}+\alpha_{\epsilon \lambda}\right\|_{\infty}<\infty \underline{\text { and }} \lim \sup _{\lambda \rightarrow 0}\left\|-\lambda^{-2} \Phi_{\epsilon \lambda}+\beta_{\epsilon \lambda}\right\|_{\infty}<\infty$

or

iii) $\lim \sup _{t \rightarrow \infty} G_{1,2}(t) / t^{q}<\infty \underline{\text { and }}$

$$
\begin{gathered}
\lim \sup _{\lambda \rightarrow 0}\left\|\sqrt{n_{\epsilon \lambda}}\left(\lambda^{-2} \Phi_{\epsilon \lambda}+g_{1}\left(n_{\epsilon \lambda}\right)+\alpha_{\epsilon \lambda}\right)\right\|_{\left(H^{1}(\Omega)\right)^{\prime}}<\infty, \\
\lim \sup _{\lambda \rightarrow 0}\left\|\sqrt{p_{\epsilon \lambda}}\left(-\lambda^{-2} \Phi_{\epsilon \lambda}+g_{2}\left(p_{\epsilon \lambda}\right)+\beta_{\epsilon \lambda}\right)\right\|_{\left(H^{1}(\Omega)\right)^{\prime}}<\infty .
\end{gathered}
$$

Then $C$ is a $\left(G_{1}, G_{2}\right)$-quantum doping profile.

Remark 1 a) The growth conditions imposed on $G_{1,2}$ in i) and iii) are rather natural in the context of deriving the Euler-Lagrange equations [7].

b) In applications pressure-functions of the types

$$
\text { (Phys) }\left\{\begin{array}{rl}
r_{1,2}(t)=O\left(t^{a}\right), r_{1,2}^{\prime}=O\left(t^{a-1}\right), & \text { as } t \rightarrow 0 \\
r_{1,2}(t)=O\left(t^{b}\right), r_{1,2}^{\prime}=O\left(t^{b-1}\right), & \text { as } t \rightarrow \infty
\end{array}, \quad \text { with } a, b \in\{1,5 / 3,7 / 3\}\right.
$$

are frequently employed. For these pressure functions we have $\lim _{t \rightarrow \infty} G(t) / t^{3}=0$. In space dimensions $d \leq 3$ we have the continuous embedding $H^{1}(\Omega) \subseteq L^{6}(\Omega)$. Hence statements i) and iii) apply in these physically relevant cases. 
Assume that $G_{1,2}$ satisfies the growth condition specified in i) and assume that $C$ is not a $\left(G_{1}, G_{2}\right)$-quantum doping profile. Then iii) has a very remarkable consequence: There is no possibility of passing to a QZSC at least weakly in $\left(H^{1}(\Omega)\right)^{\prime}$. Furthermore, the shifted electric potentials $\lambda^{-2} \Phi_{\epsilon \lambda}+\alpha_{\epsilon \lambda},-\lambda^{-2} \Phi_{\epsilon \lambda}+\beta_{\epsilon \lambda}$ which arise in equations (1) are not simultaneously uniformly bounded in $L^{\infty}(\Omega)$ as $\lambda \rightarrow 0$. These facts are in striking contrast to semi-classical settings, compare $[2,3]$.

For $\left(G_{1}, G_{2}\right)$-quantum doping profiles we can improve Theorem 1:

Theorem 3 Assume (A). Let $C$ be a $\left(G_{1}, G_{2}\right)$-quantum doping profile. Then for all $\epsilon \in] 0, \infty[:$

a) The quantum zero-space-charge energy functional $\mathcal{F}_{\epsilon}=\epsilon^{2} \mathcal{E}_{q u}+\mathcal{E}_{i}$ has a unique minimizer $\left(n_{\epsilon \circ}, p_{\epsilon \circ}\right)$ in

$$
\mathcal{C}_{\circ}=\mathcal{C} \cap\left\{(n, p) \in L^{1}(\Omega) \times L^{1}(\Omega): n-p-C=0\right\} .
$$

b) The ground energies $E_{\epsilon \lambda}$ converge to the ground energy of the quantum zero-spacecharge energy functional as $\lambda \rightarrow 0: \lim _{\lambda \rightarrow 0} E_{\epsilon \lambda}=E_{\epsilon}=\mathcal{F}_{\epsilon}\left(n_{\epsilon \circ}, p_{\epsilon \circ}\right)$.

c) $G_{1}\left(n_{\epsilon \lambda}\right) \rightarrow G_{1}\left(n_{\epsilon \circ}\right), G_{2}\left(p_{\epsilon \lambda}\right) \rightarrow G_{2}\left(p_{\epsilon \circ}\right)$ strongly in $L^{1}(\Omega)$ as $\lambda \rightarrow 0$.

d) $\lambda^{-1} \Phi_{\epsilon \lambda} \rightarrow 0, \sqrt{n_{\epsilon \lambda}} \rightarrow \sqrt{n_{\epsilon \circ}}, \sqrt{p_{\epsilon \lambda}} \rightarrow \sqrt{p_{\epsilon \circ}}$ strongly in $H^{1}(\Omega)$ as $\lambda \rightarrow 0$.

The derivation of the Euler-Lagrange equations by the standard procedure of taking directional derivatives of $\mathcal{F}_{\epsilon}$ at $\left(n_{\epsilon \circ}, p_{\epsilon \circ}\right)$ meets some difficulties.

1) If $\underline{g_{j}}=-\infty, j \in\{1,2\}$, then $G_{j}$ is not differentiable at 0 . It will however turn out that this specific inconvenience can be handled whenever ${ }^{4}$

(B.1) $\lim _{t \rightarrow 0+} \sqrt{t} g_{1,2}(t)=c_{1,2} \in \mathbb{R}$.

The physically relevant cases (Phys) are included.

2) We observe that neither $\left\|n_{\epsilon \lambda}\right\|_{\infty}$ nor $\left\|p_{\epsilon \lambda}\right\|_{\infty}$ may remain bounded as $\lambda \rightarrow 0$ :

Lemma 5 Assume (A). Let $\epsilon \in] 0, \infty\left[\right.$. Let $C$ be a $\left(G_{1}, G_{2}\right)$-quantum doping profile. Then $C \notin H^{1}(\Omega)$ implies: $\lim _{\lambda \rightarrow 0}\left\|n_{\epsilon \lambda}\right\|_{\infty}=\lim _{\lambda \rightarrow 0}\left\|p_{\epsilon \lambda}\right\|_{\infty}=\infty$.

By Lemma 5 and Theorem 3 it may happen that $n_{\epsilon \circ} \notin L^{\infty}(\Omega)$ or $p_{\epsilon \circ} \notin L^{\infty}(\Omega)$. Hence the internal energy $\mathcal{E}_{i}$ may not be differentiable at $\left(n_{\epsilon \circ}, p_{\epsilon \circ}\right)$ unless $G_{1,2}$ satisfies some growth condition, compare [7]. As we shall see soon in space dimension $d \leq 3$ the condition

(B.2) $g_{1,2}(t)=O(\ln (t))$ as $t \rightarrow \infty$ or $g_{1,2}(t)=O\left(t^{\eta}\right)$ as $t \rightarrow \infty$ with $0 \leq \eta \leq \frac{9}{2}$.

is sufficient. Again the physically relevant pressure functions of the type (Phys) are included.

3) The side constraints imposed by the set $\mathcal{C}_{\circ}$ in which $\mathcal{F}_{\epsilon}$ is to be minimized are rather restrictive. It is therefore a cumbersome task to construct curves $\gamma:[0,1] \rightarrow \mathcal{C}_{\circ}, \gamma(0)=$ $\left(n_{\epsilon \circ}, p_{\epsilon \circ}\right)$ along which the directional derivatives can be taken. A more convenient way to get the Euler-Lagrange equations of $\mathcal{F}_{\epsilon}$ is to pass to the limit $\lambda \rightarrow 0$ in the Euler-Lagrange equations of $\mathcal{E}_{\epsilon \lambda}$.

\footnotetext{
${ }^{4}$ We note that due to $g_{1,2} \in L_{l o c}^{1}\left(\left[0, \infty\left[: \lim \sup _{t \rightarrow 0} t g_{1,2}(t)=0\right.\right.\right.$.
} 
Theorem 4 Assume (A), (B.1) and (B.2). Let $\epsilon \in] 0, \infty\left[\right.$. Let $C$ be a $\left(G_{1}, G_{2}\right)$ quantum doping profile. Then there exists $\gamma_{\epsilon} \in \mathbb{R}$ such that for all $\phi \in W^{1, \infty}(\Omega)$ :

$\epsilon^{2} \int_{\Omega} \nabla \sqrt{n_{\epsilon \circ} p_{\epsilon \circ}} \cdot \nabla \phi+2 \epsilon^{2} \int_{\Omega}\left(\nabla \sqrt{n_{\epsilon \circ}} \cdot \nabla \sqrt{p_{\epsilon \circ}}\right) \phi$

$$
=-\int_{\Omega} \sqrt{n_{\epsilon \circ} p_{\epsilon \circ}}\left(g_{1}\left(n_{\epsilon \circ}\right)+g_{2}\left(p_{\epsilon \circ}\right)+\gamma_{\epsilon}\right) \phi
$$

i.e. $\left(n_{\epsilon \circ}, p_{\epsilon \circ}\right)$ is a weak solution of the quantum zero-space-charge model

$$
\begin{gathered}
\epsilon^{2}\left(\sqrt{p_{\epsilon \circ}} \Delta \sqrt{n_{\epsilon \circ}}+\sqrt{n_{\epsilon \circ}} \Delta \sqrt{p_{\epsilon \circ}}\right)=\sqrt{n_{\epsilon \circ} p_{\epsilon \circ}}\left(g_{1}\left(n_{\epsilon \circ}\right)+g_{2}\left(p_{\epsilon \circ}\right)+\gamma_{\epsilon}\right), \\
n_{\epsilon \circ}-p_{\epsilon \circ}=C .
\end{gathered}
$$

Furthermore, for all $\phi \in H^{1}(\Omega)$ :

$$
\begin{aligned}
& \lim _{\lambda \rightarrow 0} \int_{\Omega} \sqrt{n_{\epsilon \lambda}}\left(\lambda^{-2} \Phi_{\epsilon \lambda}+\alpha_{\epsilon \lambda}\right) \phi=-\epsilon^{2} \int_{\Omega} \nabla \sqrt{n_{\epsilon \circ}} \cdot \nabla \phi-\epsilon^{2} \int_{\Omega} \sqrt{n_{\epsilon \circ}} g_{1}\left(n_{\epsilon \circ}\right) \phi \\
& \lim _{\lambda \rightarrow 0} \int_{\Omega} \sqrt{p_{\epsilon \lambda}}\left(-\lambda^{-2} \Phi_{\epsilon \lambda}+\beta_{\epsilon \lambda}\right) \phi=-\epsilon^{2} \int_{\Omega} \nabla \sqrt{p_{\epsilon \circ}} \cdot \nabla \phi-\epsilon^{2} \int_{\Omega} \sqrt{p_{\epsilon \circ}} g_{2}\left(p_{\epsilon \circ}\right) \phi .
\end{aligned}
$$

Remark 2 The proof of Theorem 4 actually requires less than (B.2), namely

$$
\lim \sup _{t \rightarrow \infty} \frac{t^{3}\left[g_{j}(t)\right]^{6}}{\left(G_{j}(t)+t^{6}\right)^{5}}<\infty \quad, \quad j \in\{1,2\}
$$

\section{The Semi-classical Limit of the Quantum Zero Space Charge Model}

When setting $\epsilon=0$ in $\mathcal{F}_{\epsilon}$ one has to minimize $\mathcal{E}_{i}$ in

$$
\mathcal{D} \equiv\left\{(n, p) \in L^{1}(\Omega) \times L^{1}(\Omega): n, p \geq 0, n-p-C=0, \int_{\Omega} n=N, \int_{\Omega} p=P\right\} .
$$

This variational problem has been analyzed in [9]:

Assume (A). Then:

a) $\mathcal{E}_{i}$ has a unique minimizer $\left(n_{\circ \circ}, p_{\circ \circ}\right)$ in $\mathcal{D}$.

b) There is $\gamma_{\circ} \in \mathbb{R}$ such that

$$
\begin{array}{lll}
g_{1}\left(n_{\circ \circ}\right)+g_{2}\left(p_{\circ \circ}\right)=\gamma_{\circ} & \text { on } & \left\{n_{\circ \circ}>0\right\} \cap\left\{p_{\circ \circ}>0\right\} \\
g_{1}\left(n_{\circ \circ}\right)+g_{2}\left(p_{\circ \circ}\right) \geq \gamma_{\circ} & \text { on } & \left\{n_{\circ \circ}=0\right\} \cup\left\{p_{\circ \circ}=0\right\}
\end{array}
$$

c) $n_{\circ \circ}, p_{\circ \circ} \in L^{\infty}(\Omega)$ and $\int_{\Omega} n_{\circ \circ} p_{\circ \circ}>0$

It is very natural to expect that

$$
\lim _{\epsilon \rightarrow 0} E_{\epsilon}=\lim _{\epsilon \rightarrow 0} \inf _{\mathcal{C}_{\circ}} \mathcal{F}_{\epsilon}=\lim _{\epsilon \rightarrow 0} \mathcal{F}_{\epsilon}\left(n_{\epsilon \circ}, p_{\epsilon \circ}\right)=\mathcal{E}_{i}\left(n_{\circ \circ}, p_{\circ \circ}\right)=\inf _{\mathcal{D}} \mathcal{E}_{i}
$$

But in general we only have 
Lemma 6 Assume (A) and let $C$ be a $\left(G_{1}, G_{2}\right)$-quantum doping profile. Then

$$
\lim _{\epsilon \rightarrow 0} E_{\epsilon}=\inf _{\mathcal{C}_{\circ}} \mathcal{E}_{i} \geq \min _{\mathcal{D}} \mathcal{E}_{i}
$$

Hence two possibilities arise:

A) $\inf _{\mathcal{C}_{\circ}} \mathcal{E}_{i}>\min _{\mathcal{D}} \mathcal{E}_{i}$. The semi-classical ground energy can not be approximated by quantum ground energies. The limiting procedure $\epsilon \rightarrow 0$ is inconsistent with the underlying quantum picture, i.e. the functional $\mathcal{E}_{i}$ carries no information about its quantum origin. One is compelled to call this phenomenon a quantum gap. In this case the semi-classical model is not justified.

B) $\inf _{\mathcal{C}_{\circ}} \mathcal{E}_{i}=\min _{\mathcal{D}} \mathcal{E}_{i}$. In this case $\left(n_{\epsilon \circ}, p_{\epsilon \circ}\right)$ actually converges to $\left(n_{\circ \circ}, p_{\circ \circ}\right)$ as $\epsilon \rightarrow 0$, see the Theorem 5 below. The analysis of this limiting procedure can however not be settled by uniform bounds of $\left\|\sqrt{n_{\epsilon \circ}}\right\|_{H^{1}(\Omega)},\left\|\sqrt{p_{\epsilon \circ}}\right\|_{H^{1}(\Omega)}$, because the limiting densities $n_{\circ \circ}, p_{\circ \circ}$ may only belong to $L^{\infty}(\Omega)$. One way of controlling $n_{\epsilon \circ}, p_{\epsilon \circ}$ is the convergence of $\mathcal{E}_{i}\left(n_{\epsilon \circ}, p_{\epsilon \circ}\right)$, but two auxiliary results are needed. The first Lemma is essentially the well-known Dunford-Pettis Theorem. For the sake of a complete presentation, a proof is given in the Appendix.

Lemma 7 Let $(S, \sigma, \mu)$ be a measure space. Let $\left(f_{n}\right)_{n \in \mathbb{N}}$ be a sequence in $L^{1}(S, \sigma, \mu)$. Let $G:[0, \infty) \rightarrow \mathbb{R}$. Then:

V1. $\mu(S)<\infty$.

V2. $L^{1}(S, \sigma, \mu)$ is separable.

V3. $G$ is bounded below and $\liminf _{t \rightarrow \infty} G(t) / t=\infty$.

V4. $\exists K \in] 0, \infty\left[\right.$ such that for all $n \in \mathbb{I N}: \int_{S} G\left(\left|f_{n}\right|\right) \leq K$.

$\Rightarrow \quad$ There exists a subsequence $\left(f_{n^{\prime}}\right)_{n^{\prime} \in \mathbb{N}^{\prime}}$ of $\left(f_{n}\right)_{n \in \mathbb{N}}$ and $f \in L^{1}(S, \sigma, \mu)$ such that

$$
f_{n^{\prime}} \rightarrow f \text { weakly in } L^{1}(S, \sigma, \mu) \text { as } n^{\prime} \rightarrow \infty .
$$

The proof of the subsequent compactness-by-convexity principle can essentially be found in [9]. We leave the minor extension of the proof to the more general measurespace setting to the reader.

Lemma 8 Let $(S, \sigma, \mu)$ be a measure space. Let $\left(f_{n}\right)_{n \in \mathbb{N}}$ be a sequence in $L^{1}(S, \sigma, \mu)$. Let $f \in L^{1}(S, \sigma, \mu)$. Let $G:[0, \infty) \rightarrow \mathbb{R}$. Then:

V1. $\mu(S)<\infty$.

V2. $\forall n \in \mathbb{I N}: f_{n} \geq 0$.

V3. $f_{n} \rightarrow f$ weakly in $L^{1}(S, \sigma, \mu)$ as $n \rightarrow \infty$.

V4. $G$ is strictly convex and continuous.

V5. $\lim _{n \rightarrow \infty} \int_{S} G\left(f_{n}\right)=\int_{S} G(f)$.

$\Rightarrow \quad f_{n} \rightarrow f$ strongly in $L^{1}(S, \sigma, \mu)$.

With the aid of Lemma 7 and Lemma 8, we can carry out the semi-classical limit of the quantum zero-space-charge model. 
Theorem 5 Assume (A) and let $C$ be a $\left(G_{1}, G_{2}\right)$-quantum doping profile. Furthermore, assume that $\inf _{\mathcal{C}_{\circ}} \mathcal{E}_{i}=\inf _{\mathcal{D}} \mathcal{E}_{i}$. Then:

A1. $n_{\epsilon \circ} \rightarrow n_{\circ \circ}, p_{\epsilon \circ} \rightarrow p_{\circ \circ}$ strongly in $L^{1}(\Omega)$ as $\epsilon \rightarrow 0$.

A2. $\left\|\sqrt{n_{\epsilon \circ}}\right\|_{H^{1}(\Omega)},\left\|\sqrt{p_{\epsilon \circ}}\right\|_{H^{1}(\Omega)}=o(1 / \epsilon)$ as $\epsilon \rightarrow 0$.

A3. If $\left(n_{\circ \circ}, p_{\circ \circ}\right) \in \mathcal{C}_{\circ}$, then

$$
\sqrt{n_{\epsilon \circ}} \rightarrow \sqrt{n_{\circ \circ}}, \sqrt{p_{\epsilon \circ}} \rightarrow \sqrt{p_{\circ \circ}} \text { strongly in } H^{1}(\Omega) \text { as } \epsilon \rightarrow 0 .
$$

\section{The limit $\epsilon=\lambda \rightarrow 0$}

By a straightforward combination of the techniques employed to prove Theorem 3 and Theorem 5 one easily establishes

Theorem 6 Assume $(\mathbf{A})$ and let $C$ be $a\left(G_{1}, G_{2}\right)$-quantum doping profile. Furthermore, assume that $\inf _{\mathcal{C}_{\circ}} \mathcal{E}_{i}=\inf _{\mathcal{D}} \mathcal{E}_{i}$. Then:

A1. $n_{\epsilon \epsilon} \rightarrow n_{\circ \circ}, p_{\epsilon \epsilon} \rightarrow p_{\circ \circ}$ strongly in $L^{1}(\Omega)$ as $\epsilon \rightarrow 0$.

A2. $\left\|\sqrt{n_{\epsilon \epsilon}}\right\|_{H^{1}(\Omega)},\left\|\sqrt{p_{\epsilon \epsilon}}\right\|_{H^{1}(\Omega)}=o(1 / \epsilon)$ as $\epsilon \rightarrow 0$.

A3. If $\left(n_{\circ \circ}, p_{\circ \circ}\right) \in \mathcal{C}_{\circ}$, then

$$
\sqrt{n_{\epsilon \epsilon}} \rightarrow \sqrt{n_{\circ \circ}}, \sqrt{p_{\epsilon \epsilon}} \rightarrow \sqrt{p_{\circ \circ}} \text { strongly in } H^{1}(\Omega) \text { as } \epsilon \rightarrow 0 .
$$

\section{Conclusions}

In this paper an asymptotic analysis of a bipolar quantum hydrodynamic model in thermal equilibrium has been performed. The investigations apply in cases where the scaled minimal Debye length $\lambda$ is at most of the order of magnitude of the scaled Planck's constant $\epsilon$.

The limit $\lambda \rightarrow 0$ leads to a physically reasonable model - the quantum zero-space-charge model - if and only if the doping profile $C$ is a $\left(G_{1}, G_{2}\right)$-quantum doping profile. Doping profiles with abrupt junctions are not $\left(G_{1}, G_{2}\right)$-quantum doping profiles. Their employment in quantum models which incorporate the zero-space-charge approximation is therefore doubtful.

Theorem 2, Lemma 1, Lemma 2, Lemma 3, Lemma 4 and Lemma 5 are concerned with several properties of $\left(G_{1}, G_{2}\right)$-quantum doping profiles. Lemma 2 and Lemma 3 include discussions of "closeness" of $\left(G_{1}, G_{2}\right)$-quantum doping profiles to $H^{1}(\Omega)$-functions. In one space dimension, $\left(G_{1}, G_{2}\right)$-quantum doping profiles and $H^{1}(\Omega)$-functions coincide. However in case of $d \geq 2$ the discussion is much more complicated (but compare Lemma 3 ). To give an impression of the problems just consider $d=2$ and assume that $C$ is a $\left(G_{1}, G_{2}\right)$-quantum doping profile. According to assumption (A) and Lemma 1 there are $f_{1}, f_{2} \in H^{1}(\Omega)$ with

$$
f_{1} f_{2} \in L^{\infty}(\Omega) \quad, \quad f_{1} \geq 0 \quad, \quad f_{2} \in L^{\infty}(\Omega) .
$$


With respect to Lemma 5 (one may expect that the particle densities are uniformly bounded as $\lambda \rightarrow 0$ ) it seems apropriate to try a verification of " $C \in H^{1}(\Omega)$ " first. But then one has to prove:

$$
\text { If } f_{1}, f_{2} \in H^{1}(\Omega), f_{1} f_{2} \in L^{\infty}(\Omega), f_{1} \geq 0, f_{2} \in L^{\infty}(\Omega) \text {, then } f_{1} \nabla f_{2} \in L^{2}\left(\Omega: \mathbb{R}^{2}\right) .
$$

The (sharp!) Sobolev imbedding Theorem assures $f_{1} \nabla f_{2} \in L^{q}\left(\Omega: \mathbb{R}^{2}\right)$ for all $q \in[1,2[$. Since $f_{1} \in L^{t}(\Omega)$ for all $t \in\left[1, \infty\left[\right.\right.$, it remains to consider the cases where $f_{1} \in H^{1}(\Omega)$, $f_{1} \notin L^{\infty}(\Omega), f_{2} \in L^{\infty}(\Omega), f_{1} f_{2} \in L^{\infty}(\Omega), \nabla f_{2} \in L^{2}\left(\Omega: \mathbb{R}^{2}\right)$ but $\nabla f_{2} \notin L^{2+\xi}\left(\Omega: \mathbb{R}^{2}\right)$ for all $\xi \in] 0, \infty\left[\right.$. These assumptions imply: For all $\left.M_{1}, M_{2} \in\right] 0, \infty\left[:\right.$ The sets $A\left(M_{1}\right):=$ $\left\{f_{1} \geq M_{1}\right\}$ and $B\left(M_{2}\right):=\left\{\left|\nabla f_{2}\right| \geq M_{2}\right\}$ have nonzero measure. Two possibilities arise: If there are $\left.M_{1}, M_{2} \in\right] 0, \infty\left[\right.$ such that the measure of $A\left(M_{1}\right) \cap B\left(M_{2}\right)$ is zero, then $f_{1} \nabla f_{2} \in L^{2}(\Omega)$. In this case we are done. The remaining - and rather complicated - situation concerns cases where the set $A\left(M_{1}\right) \cap B\left(M_{2}\right)$ has nonzero measure for all $\left.M_{1}, M_{2} \in\right] 0, \infty$. The assumption $f_{1} f_{2} \in L^{\infty}(\Omega)$ only implies that $f_{2}$ has to become smaller and smaller on $A\left(M_{1}\right) \cap B\left(M_{2}\right)$ as $M_{1}$ tends to infinity, but from this one can get no information about the behaviour of $\nabla f_{2}$.

In this phase of the investigations some doubts may arise whether each $\left(G_{1}, G_{2}\right)$-quantum doping profile is in $H^{1}(\Omega)$ and one may think of constructing a counter example ${ }^{5}$. The previous investigations show that one has to find $f_{1}, f_{2} \in H^{1}(\Omega)$ with $f_{1} \geq 0, f_{2} \in L^{\infty}(\Omega)$, $f_{1} f_{2} \in L^{\infty}(\Omega)$ and $f_{1} \nabla f_{2} \notin L^{2}\left(\Omega: \mathbb{R}^{2}\right)$. Then one has to look for $f_{1} \notin L^{\infty}(\Omega)$ and $\nabla f_{2} \in L^{2}\left(\Omega: \mathbb{R}^{2}\right)$ but $\nabla f_{2} \notin L^{2+\xi}\left(\Omega: \mathbb{R}^{2}\right)$ for all $\left.\xi \in\right] 0, \infty\left[\right.$. Standard functions $f_{1}, f_{2}$ satisfying the last two conditions are well-known - but just these two conditions do not imply $f_{1} \nabla f_{2} \notin L^{2}(\Omega)$. Indeed, if - as above - the sets where $f_{1}$ and $\nabla f_{2}$ are "infinite" are separated, then $f_{1} \nabla f_{2} \in L^{2}(\Omega)$. If these singular sets intersect, then $f_{2}$ has to tend as fast to zero as $f_{1}$ tends to infinity to ensure that $f_{1} f_{2} \in L^{\infty}(\Omega)$. Considering the standard example $f_{1}=|\ln | \ln (r)||$, this requirement is in competition with the orders of growth of $f_{1}$ and $\nabla f_{2}$ (the well-known examples for $f_{2}$ involve hard-to-estimate infinite series).

For $\left(G_{1}, G_{2}\right)$-quantum doping profiles the quantum zero-space-charge model is a reasonable approximation in cases where $\lambda^{2} \ll \epsilon^{2}$. If additionally $\epsilon^{2} \ll 1$ holds, then the subsequent limit $\epsilon \rightarrow 0$ (performed in Section 3) will be of interest. It can not be excluded that a "quantum gap" appears, i.e. the limiting energy (as $\epsilon \rightarrow 0$ ) is less than the energies of the approximating quantum models. A discussion of this phenomenon may be helpful ${ }^{6}$.

1. If $\left(n_{\circ \circ}, p_{\circ \circ}\right) \in \mathcal{C}_{\circ}$, then trivially $\inf _{\mathcal{C}_{\circ}} \mathcal{E}_{i} \leq \min _{\mathcal{D}} \mathcal{E}_{i}=\mathcal{E}_{i}\left(n_{\circ \circ}, p_{\circ \circ}\right)$ and no quantum gap can arise.

2. The condition of 1 . applies in cases where $n_{\circ \circ}, p_{\circ \circ} \in H^{1}(\Omega)$ with $n_{\circ \circ}, p_{\circ \circ} \geq \rho$, $\rho \in] 0, \infty\left[\right.$, because then $\sqrt{n_{\circ \circ}} \in H^{1}(\Omega)$ and $\sqrt{p_{\circ \circ}} \in H^{1}(\Omega)$ and therefore $\left(n_{\circ \circ}, p_{\circ \circ}\right) \in$ $\mathcal{C}_{0}$.

3. It is easy to see that a quantum gap will appear if and only if for all sequences $\left(n_{k}, p_{k}\right)_{k \in \mathbb{N}}$ in $\mathcal{C}_{\circ}$ we have

$$
\liminf _{k \rightarrow \infty} \mathcal{E}_{i}\left(n_{k}, p_{k}\right)>\mathcal{E}_{i}\left(n_{\circ \circ}, p_{\circ \circ}\right) .
$$

\footnotetext{
${ }^{5} \mathrm{~A}$ counter example may be easier to find in dimension $d=3$, but the problems are the same as for $d=2$.

${ }^{6}$ Notations as in Sections 2 and 3.
} 
4. Let us consider the cases $C \in H^{1}(\Omega)$ and

$$
r(t)=r_{1,2}(t)=t^{a} \quad, \quad a \in[1, \infty[
$$

now.

a) In the case $a=1$, the reader will have no difficulties to deduce from the auxiliary result at the beginning of Section 3 that

$$
n_{\circ \circ}=\frac{1}{2}\left(C+\sqrt{C^{2}+4 \rho^{2}}\right), p_{\circ \circ}=\frac{1}{2}\left(-C+\sqrt{C^{2}+4 \rho^{2}}\right),
$$

where $\rho \in] 0, \infty[$. Due to the discussion of 2 . no quantum gap can arise.

b) In the case $a>1$ there exists, due to the auxiliary result at the beginning of Section 3 , a $\rho \in] 0, \infty[$ with

$$
C=n_{\circ \circ}-p_{\circ \circ}
$$

and

$$
\begin{aligned}
& n_{\circ \circ}^{\alpha}+p_{\circ \circ}^{\alpha}=\rho^{2} \quad \text { on } \quad\left\{n_{\circ \circ}>0\right\} \cap\left\{p_{\circ \circ}>0\right\} . \\
& n_{\circ \circ}^{\alpha}+p_{\circ \circ}^{\alpha} \geq \rho^{2} \quad \text { on } \quad\left\{n_{\circ \circ}=0\right\} \cup\left\{p_{\circ \circ}=0\right\}
\end{aligned}
$$

where $\alpha:=a-1 \in] 0, \infty\left[\right.$. We get an algebraic equation for $p_{\circ \circ}$ :

$$
\Phi\left(p_{\circ \circ}\right):=p_{\circ \circ}-\left(\left[\rho^{2}-p_{\circ \circ}^{\alpha}\right]^{+}\right)^{1 / \alpha}=C .
$$

It is easy to see that $\Phi$ is strictly increasing on $\left[0, \infty\left[. \Phi^{-1}:\left[-\rho^{2 / \alpha}, \infty[\rightarrow[0, \infty[\right.\right.\right.$ is uniformly Lipschitz-continuous. Since $C \in H^{1}(\Omega) \cap L^{\infty}(\Omega)$ we get $p_{\circ \circ} \in H^{1}(\Omega)$ as well as $n_{\circ \circ} \in H^{1}(\Omega)$ and we conclude with the aid of 2: If $n_{\circ \circ}, p_{\circ \circ}$ are uniformly bounded below by a positive constant, then there will be no quantum gap.

c) When is a quantum gap in case of $a>1$ possible? With respect to b) the appearance of vacuum sets (subsets of $\Omega$ where $n_{\circ \circ}$ or $p_{\circ \circ}$ vanishes) is a good starting point ${ }^{7}$. Furthermore, $\sqrt{n_{\circ \circ}}$ (or $\sqrt{p_{\circ \circ}}$ ) does not belong to $H^{1}(\Omega)$ (this is, e.g., the case when $n_{\circ \circ}$ is piecewise affine with a vacuum set of nonzero measure). Finally it is not possible (and this is the least transparent condition) to approximate $\sqrt{n_{\circ \circ}}$ and $\sqrt{p_{\circ \circ}}$ by non-negative functions $n_{k}$ and $p_{k}$ such that $\left(n_{k}, p_{k}\right)_{k \in \mathbb{N}}$ is a sequence in $\mathcal{C}_{\mathrm{o}}$ with $\lim _{k \rightarrow \infty}\left\|n_{k}-n_{\circ \circ}\right\|_{L^{a}(\Omega)}+\left\|p_{k}-p_{\circ \circ}\right\|_{L^{a}(\Omega)}=0$. Indeed: an approximation of $n_{\circ \circ}$ and $p_{\circ \circ}$ by non-negative functions $n_{k}$ and $p_{k}$, respectively, whose square-root belongs to $H^{1}(\Omega)$ and which converge strongly in $L^{a}(\Omega)$ to $n_{\circ \circ}$ and $p_{\circ \circ}$, respectively, is always possible - but then it has to be expected that $n_{k}, p_{k}$ violate the additional requirements

$$
C=n_{k}-p_{k}=n_{\circ \circ}-p_{\circ \circ} \quad, \quad \int_{\Omega} n_{k}=\int_{\Omega} n_{\circ \circ}, \int_{\Omega} p_{k}=\int_{\Omega} p_{\circ \circ}
$$

for all $k \in \mathbb{I}$, i.e. $\left(n_{k}, p_{k}\right) \notin \mathcal{C}_{\circ}$. This observation makes it plausible to expect quantum gaps in certain situations.

In Section 4 the simultaneous limit $\epsilon=\lambda \rightarrow 0$ is considered. The limiting model coincides with the classical model whenever $C$ is a $\left(G_{1}, G_{2}\right)$-quantum doping profile and whenever no quantum gap appears.

\footnotetext{
${ }^{7}$ Such thermal equilibrium densities actually exist, see [4], [8].
} 


\section{A Proofs}

\section{Proof of Theorem 1:}

We observe that $\left(n_{\epsilon \lambda}, p_{\epsilon \lambda}\right)$ is the unique minimizer of $\lambda^{2} \mathcal{E}_{\epsilon \lambda}=\lambda^{2} \epsilon^{2} \mathcal{E}_{q u}+\lambda^{2} \mathcal{E}_{i}+\mathcal{E}_{e l}$. Given $\delta>0$ choose a $H^{1}(\Omega)$-regularizer $\left(n^{\delta}, p^{\delta}\right) \in \mathcal{C} \cap$ $\left(L^{\infty}(\Omega) \times L^{\infty}(\Omega)\right)$ of $\left(C^{+}+\left(\rho_{i} / \operatorname{meas}(\Omega)\right), C^{-}+\left(\rho_{i} / \operatorname{meas}(\Omega)\right)\right)$ with $\left\|n^{\delta}-p^{\delta}-C\right\|_{2} \leq \delta$. Set $\Phi^{\delta}=\Phi\left[n^{\delta}-p^{\delta}-C\right]$. Then there exists due to Poincaré's inequality a $\left.K \in\right] 0, \infty[$ (independently of $\epsilon, \lambda$ ) such that

$$
\mathcal{E}_{e l}\left(n^{\delta}, p^{\delta}\right)=\frac{1}{2}\left\|\nabla \Phi^{\delta}\right\|_{2}^{2} \leq K\left\|n^{\delta}-p^{\delta}-C\right\|_{2}^{2} \leq K \delta^{2} .
$$

Hence

$$
\begin{aligned}
0 \leq \limsup _{\lambda \rightarrow 0} \lambda^{2} E_{\epsilon \lambda} & \leq \lim _{\lambda \rightarrow 0} \lambda^{2} \mathcal{E}_{\epsilon \lambda}\left(n^{\delta}, p^{\delta}\right) \\
& =\lim _{\lambda \rightarrow 0} \lambda^{2} \epsilon^{2} \mathcal{E}_{q u}\left(n^{\delta}, p^{\delta}\right)+\lambda^{2} \mathcal{E}_{i}\left(n^{\delta}, p^{\delta}\right)+\mathcal{E}_{e l}\left(n^{\delta}, p^{\delta}\right) \\
& =\mathcal{E}_{e l}\left(n^{\delta}, p^{\delta}\right) \leq K \delta^{2}
\end{aligned}
$$

Since $\delta \in] 0, \infty\left[\right.$ was arbitrary it follows that $\lim _{\lambda \rightarrow 0} \lambda^{2} E_{\epsilon \lambda}=\lim _{\lambda \rightarrow 0} \mathcal{E}_{\epsilon \lambda}\left(n_{\epsilon \lambda}, p_{\epsilon \lambda}\right)=0$. This settles c). Furthermore, we have shown that $\lim _{\lambda \rightarrow 0} \mathcal{E}_{e l}\left(n_{\epsilon \lambda}, p_{\epsilon \lambda}\right)=0$, which proves a). Concerning b) let $\phi \in H^{1}(\Omega)$. Then $\int_{\Omega}\left(n_{\epsilon \lambda}-p_{\epsilon \lambda}-C\right) \phi=\int_{\Omega} \nabla \Phi_{\epsilon \lambda} \cdot \nabla \phi$. Hence b) follows from a).

$\square$. Proof of Theorem 2:

i) $\Rightarrow$ ii) is clear. ii) $\Rightarrow$ iii): By assumption there are $\epsilon \in] 0, \infty[$ and $K \in] 0, \infty[$ such that for all $\lambda \in] 0, \infty[$ :

$$
\left\|\nabla \sqrt{n_{\epsilon}}\right\|_{2},\left\|\nabla \sqrt{p_{\epsilon \lambda}}\right\|_{2}, \mathcal{E}_{i}\left(n_{\epsilon \lambda}, p_{\epsilon \lambda}\right)<K
$$

Due to $\left\|\sqrt{n_{\epsilon \lambda}}\right\|_{2},\left\|\sqrt{p_{\epsilon \lambda}}\right\|_{2} \leq N+P$ we have by passing to a subnet: There exists $\left(n^{*}, p^{*}\right) \in$ $\mathcal{C}$ such that

$$
\begin{gathered}
\sqrt{n_{\epsilon \lambda}} \rightarrow \sqrt{n^{*}}, \sqrt{p_{\epsilon \lambda}} \rightarrow \sqrt{p^{*}} \text { weakly in } H^{1}(\Omega) \text { as } \lambda \rightarrow 0, \\
n_{\epsilon \lambda} \rightarrow n^{*}, p_{\epsilon \lambda} \rightarrow p^{*} \text { strongly in } L^{2}(\Omega) \text { as } \lambda \rightarrow 0 .
\end{gathered}
$$

Due to Theorem 1 we have $n^{*}-p^{*}-C=0$ and by weak lower semi-continuity we have $\mathcal{E}_{i}\left(n^{*}, p^{*}\right) \leq K<\infty$.

iii) $\Rightarrow$ i): Since $\Phi\left[n^{*}-p^{*}-C\right]=0$, we have for all $\left.\epsilon, \lambda \in\right] 0, \infty[$

$$
E_{\epsilon \lambda} \leq \mathcal{E}_{\epsilon \lambda}\left(n^{*}, p^{*}\right)=\epsilon^{2} \mathcal{E}_{q u}\left(n^{*}, p^{*}\right)+\mathcal{E}_{i}\left(n^{*}, p^{*}\right)=K_{\epsilon}<\infty
$$

where $\left.K_{\epsilon} \in\right] 0, \infty[$ depends on $\epsilon$ but is independent of $\lambda$.

\section{Proof of Lemma 1:}

i) $\Rightarrow$ ii): We have $C=|r|^{2}-|s|^{2}$ where $|r|,|s| \in H^{1}(\Omega)$, since $|\cdot|$ maps $H^{1}(\Omega)$ into $H^{1}(\Omega)$. Hence we may assume without loss of generality that $r, s \geq 0$. Let $f_{1}=r+s$ and $f_{2}=r-s$. Then $C=f_{1} f_{2}$ and $f_{1}, f_{2} \in H^{1}(\Omega)$ and $f_{1} \geq 0$. It remains to be shown that $f_{2}=r-s \in L^{\infty}(\Omega)$. Let $K>\sqrt{\|C\|_{\infty}}$. Then for all $x \in \Omega_{K}=\left\{f_{2} \geq K\right\}$ : $C(x)=f_{1}(x) f_{2}(x)=(r+s)(x)(r-s)(x) \geq(K+2 s(x)) K \geq K^{2}>\|C\|_{\infty}$. Henc meas $\left(\Omega_{K}\right)=0$ and therefore $\left\|f_{2}\right\|_{\infty} \leq K$.

ii) $\Rightarrow$ i): Set $r=\left(f_{1}+f_{2}\right) / 2$ and $s=\left(f_{1}-f_{2}\right) / 2$.

\section{Proof of Lemma 2:}

2: If $C \in H^{1}(\Omega)$, then $C^{+}+1 \in H^{1}(\Omega)$ and $C^{-}+1 \in H^{1}(\Omega)$ as well as $r:=\sqrt{C^{+}+1} \in$ 
$H^{1}(\Omega)$ and $s:=\sqrt{C^{-}+1} \in H^{1}(\Omega)$. In this case we have $C=r^{2}-s^{2}$ with $r, s \in$ $H^{1}(\Omega) \cap L^{\infty}(\Omega)$. Hence $\int_{\Omega} G_{1}\left(r^{2}\right)<\infty$ and $\int_{\Omega} G_{2}\left(s^{2}\right)<\infty$. This shows that $C$ is a $\left(G_{1}, G_{2}\right)$-quantum doping profile.

1: Because of 2 . it sufficies to prove that each $\left(G_{1}, G_{2}\right)$-quantum doping profile is in $H^{1}(\Omega)$. Since $d=1$ we have the inclusion $H^{1}(\Omega) \subseteq L^{\infty}(\Omega)$, see [1]. On the other hand we have $C=r^{2}-s^{2}$ and therefore $C \in W^{1,1}(\Omega)$, which implies [11] that $C^{\prime}=2 r r^{\prime}-2 s s^{\prime}$. Since $r^{\prime}, s^{\prime} \in L^{2}(\Omega)$ and $r, s \in L^{\infty}(\Omega)$ we get $C^{\prime} \in L^{2}(\Omega)$ and therefore $C \in H^{1}(\Omega)$.

3: If $C$ is a $\left(G_{1}, G_{2}\right)$-quantum doping profile, then $C=r^{2}-s^{2}$ for some $r, s \in H^{1}(\Omega)$. According to [1] we have $r, s \in L^{t}(\Omega)$ for all $t \in\left[1, \infty\left[\right.\right.$. Furthermore, $r^{2}, s^{2} \in W^{1,1}(\Omega)$ and therefore $C \in W^{1,1}(\Omega)$. Hence $\nabla C=2 r \nabla r-2 s \nabla s$. Since $r, s \in L^{t}(\Omega)$ for all $t \in\left[1, \infty\left[\right.\right.$ and since $\nabla r, \nabla s \in L^{2}\left(\Omega: \mathbb{R}^{2}\right)$, we get from Hölder's inequality $\nabla C \in L^{\frac{2 t}{t+2}}(\Omega)$ for all $t \in\left[2, \infty\left[\right.\right.$, i.e. $\nabla C \in L^{q}(\Omega)$ for all $q \in[1, \infty[$.

4. follows by an argument similiar to the proof of 3 . by employing $H^{1}(\Omega) \subseteq L^{6}(\Omega)$ in case of $d=3$.

\section{Proof of Lemma 3:}

By Lemma 1: There are $f_{1}, f_{2} \in H^{1}(\Omega)$ such that $C=f_{1} f_{2}, f_{1} \geq 0, f_{2} \in L^{\infty}(\Omega)$. By standard properties of Sobolev functions (see e.g. [11]) there are representatives $\overline{f_{i}}$ of $f_{i}$, $i \in\{1,2\}$, such that for almost all $\vec{v} \in \vec{\xi}^{\perp}$ with $I(\vec{\xi}, \vec{v}) \neq \emptyset$ : The mapping

$$
\begin{aligned}
\overline{f_{i}}(\vec{v}+[.] \vec{\xi}): I(\vec{\xi}, \vec{v}) & \rightarrow \mathbb{R} \\
t & \mapsto \overline{f_{i}}(\vec{v}+t \vec{\xi})
\end{aligned}
$$

is absolutely continuous, see e.g. [6]. We can assume without loss of generality: $\overline{f_{1}} \geq 0$ and $\overline{f_{2}}$ is bounded. Hence (see again [6]) for almost all $\vec{v} \in \vec{\xi}^{\perp}$ with $I(\vec{\xi}, \vec{v}) \neq \emptyset$ and almost all $t \in I(\vec{\xi}, \vec{v}): \overline{f_{i}}(\vec{v}+[.] \vec{\xi})$ is differentiable at $t$, the respective derivation ${\overline{f_{i}}}^{\prime}(\vec{v}+t . \vec{\xi}):=$ ${\overline{f_{i}}}^{\prime}(\vec{v}+[.] \vec{\xi})(t)$ is Lebesgue integrable on $I(\vec{\xi}, \vec{v})$ and the integration by parts formula holds, where $i \in\{1,2\}$. From these properties of $\overline{f_{i}}(\vec{v}+[.] \vec{\xi}), i \in\{1,2\}$ it is easy to deduce that for almost all $\vec{v} \in \vec{\xi}^{\perp}$ with $I(\vec{\xi}, \vec{v}) \neq \emptyset$ : $\overrightarrow{f_{i}}(\vec{v}+[.] \vec{\xi})$ belongs to $W^{1,1}(I(\vec{v}, \vec{\xi})$ ) (where ${\overline{f_{i}}}^{\prime}(\vec{v}+[.] \vec{\xi})$ coincides with its distributional derivative). Now we shall prove: For almost all $\vec{v} \in \vec{\xi}^{\perp}$ with $I(\vec{\xi}, \vec{v}) \neq \emptyset: \vec{f}_{i}^{\prime}(\vec{v}+[.] \vec{\xi}) \in L^{2}(I(\vec{\xi}, \vec{v}))$. Let $u_{1,2}$ be representatives of the directional derivations $\vec{\xi} \cdot \nabla f_{1,2}$, respectively. Then we have for all $\phi \in C_{c}^{\infty}(\Omega)$ by Fubini's Theorem for $i \in\{1,2\}$ the identities

$$
\int_{\Omega} f_{i}(\vec{\xi} \cdot \nabla \phi)=-\int_{\Omega} u_{i} \phi=-\int_{\vec{\xi}^{\perp}} \int_{I(\vec{\xi}, \vec{v})} u_{i}(\vec{v}+t \vec{\xi}) \phi(\vec{v}+t \vec{\xi})
$$

while on the other hand

$$
\int_{\Omega} f_{i}(\vec{\xi} \cdot \nabla \phi)=\int_{\vec{\xi}^{\perp}} \int_{I(\vec{\xi}, \vec{v})} \overline{f_{i}}(\vec{v}+t \vec{\xi})(\vec{\xi} \cdot \nabla \phi)(\vec{v}+t \vec{\xi})=-\int_{\vec{\xi}^{\perp}} \int_{I(\vec{\xi}, \vec{v})}{\overline{f_{i}}}^{\prime}(\vec{v}+t \vec{\xi}) \phi(\vec{v}+t \vec{\xi}) .
$$

From these two identities we get by a standard density argument for almost all $\vec{v} \in \vec{\xi}^{\perp}$ with $I(\vec{\xi}, \vec{v}) \neq \emptyset$ : There is a nullset $N_{\vec{v}} \subseteq I(\vec{\xi}, \vec{v})$ such that $u_{i}(\vec{v}+t \vec{\xi})={\overrightarrow{f_{i}}}^{\prime}(\vec{v}+t \vec{\xi})$ for all $t \in$ $I(\vec{\xi}, \vec{v}) \backslash N_{\vec{v}}$. While this result does not imply that the mapping $\Omega \ni \vec{x} \mapsto \bar{f}_{i}^{\prime}(\vec{v}(\vec{x})+t(\vec{x}) \vec{\xi})$ is measurable, we nevertheless have for almost all $\vec{v} \in \vec{\xi}^{\perp}$ with $I(\vec{\xi}, \vec{v}) \neq \emptyset$ and $i \in\{1,2\}$ :

$$
\int_{I(\vec{\xi}, \vec{v})}\left|{\overrightarrow{f_{i}}}^{\prime}(\vec{v}+t \vec{\xi})\right|^{2}=\int_{I(\vec{\xi}, \vec{v})}\left|u_{i}(\vec{v}+t \vec{\xi})\right|^{2} \in[0, \infty] .
$$

On the other hand, $u_{i}$ is for $i \in\{1,2\}$ square integrable over $\Omega$. Hence - again by Fubini's Theorem -

$$
\int_{\vec{\xi}^{\perp}} \int_{I(\vec{\xi}, \vec{v})}\left|u_{i}(\vec{v}+t \vec{\xi})\right|^{2}=\int_{\Omega}\left|u_{i}\right|^{2}<\infty
$$


and therefore for almost all $\vec{v} \in \vec{\xi}^{\perp}$ :

$$
\int_{I(\vec{\xi}, \vec{v})}\left|u_{i}(\vec{v}+t \vec{\xi})\right|^{2}<\infty
$$

We conclude: for almost all $\vec{v} \in \vec{\xi}^{\perp}$ with $I(\vec{\xi}, \vec{v}) \neq \emptyset$ and $i \in\{1,2\}$ :

$$
{\overline{f_{i}}}^{\prime}(\vec{v}+[.] \vec{\xi}) \in L^{2}(I(\vec{\xi}, \vec{v}))
$$

and therefore

$$
\overline{f_{i}}(\vec{v}+[.] \vec{\xi}) \in H^{1}(I(\vec{\xi}, \vec{v})) .
$$

$\overline{f_{1} f_{2}}$ is a representative of $C$. We set $c:=\overline{f_{1} f_{2}}$ and get for almost all $\vec{v} \in \vec{\xi}^{\perp}$ with $I(\vec{\xi}, \vec{v}) \neq \emptyset$ and $i \in\{1,2\}$ : The one-dimensional function $c(\vec{v}+[.] \vec{\xi})$ is the product of two one-dimensional $H^{1}(I(\vec{\xi}, \vec{v}))$ functions $\overline{f_{1}}$ and $\overline{f_{2}}$, where the first function is non-negative and the second one belongs to $L^{\infty}(I(\vec{\xi}, \vec{v}))$. By Lemma 1: $c$ is a $\left(G_{1}, G_{2}\right)$-quantum doping profile and it readily follows from Lemma 2: $c \in H^{1}(I(\vec{\xi}, \vec{v}))$.

\section{Proof of Lemma 4:}

i) By assumption the $H^{1}(\Omega)$-norms of $\sqrt{n_{\epsilon \lambda}}, \sqrt{p_{\epsilon \lambda}}$ are bounded as $\lambda \rightarrow 0$. Hence the internal energy $\mathcal{E}_{i}$ is due to the assumptions on $G_{1,2}$ bounded, too. By weak $H^{1}(\Omega)$ convergence of a subnet of $\left(\sqrt{n_{\epsilon \lambda}}, \sqrt{p_{\epsilon \lambda}}\right)$ we have due to Theorem 1 that $C$ is the difference of squares of $H^{1}(\Omega)$-functions whose internal energy is by weak convergence bounded. The result follows from Theorem 2 .

ii) Due to the maximum principle and $\overline{g_{1,2}}=\infty$, the $L^{\infty}(\Omega)$-norms of $\sqrt{n_{\epsilon \lambda}}, \sqrt{p_{\epsilon \lambda}}$ are bounded. It is also easy to see that the right-hand sides of equations (1) are uniformly bounded in $L^{\infty}(\Omega)$. Therefore, the $H^{1}(\Omega)$-norms of $\sqrt{n_{\epsilon \lambda}}, \sqrt{p_{\epsilon \lambda}}$ are uniformly bounded. The result now follows similar as in i), since the internal energy is due to the uniform $L^{\infty}(\Omega)$-bounds of $\sqrt{n_{\epsilon}}, \sqrt{p_{\epsilon \lambda}}$ uniformly bounded, too.

iii) Let $A_{\epsilon}(\lambda) \equiv \sqrt{n_{\epsilon \lambda}}\left(\lambda^{-2} \Phi_{\epsilon \lambda}+g_{1}\left(n_{\epsilon \lambda}\right)+\alpha_{\epsilon \lambda}\right)$ and let $\phi \in H^{1}(\Omega)$. Then we have $\left\langle A_{\epsilon}(\lambda) \mid \phi\right\rangle=-\epsilon \int_{\Omega} \nabla \sqrt{n_{\epsilon \lambda}} \cdot \nabla \phi$. Hence for all $\left.\lambda \in\right] 0, \infty[$,

$$
\begin{aligned}
\left\|\sqrt{n_{\epsilon \lambda}}\right\|_{H^{1}(\Omega)} & =\sup _{\|\phi\|_{H^{1}(\Omega)}=1}\left|\int_{\Omega} \nabla \sqrt{n_{\epsilon \lambda}} \cdot \nabla \phi+\int_{\Omega} \sqrt{n_{\epsilon \lambda}} \phi\right| \\
& \leq(1 / \epsilon)\left\|A_{\epsilon}(\lambda)\right\|_{\left(H^{1}(\Omega)\right)^{\prime}}+\sup _{\|\phi\|_{H^{1}(\Omega)}=1}\left\|\sqrt{n_{\epsilon \lambda}}\right\|_{2}\|\phi\|_{2} \\
& =(1 / \epsilon)\left\|A_{\epsilon}(\lambda)\right\|_{\left(H^{1}(\Omega)\right)^{\prime}}+\sqrt{N} .
\end{aligned}
$$

In analogy we get the estimate $\left\|\sqrt{p_{\epsilon \lambda}}\right\|_{H^{1}(\Omega)} \leq(1 / \epsilon)\left\|B_{\epsilon}(\lambda)\right\|_{\left(H^{1}(\Omega)\right)^{\prime}}+\sqrt{P}$, where $B_{\epsilon}(\lambda) \equiv \sqrt{p_{\epsilon \lambda}}\left(-\lambda^{-2} \Phi_{\epsilon \lambda}+g_{2}\left(p_{\epsilon \lambda}\right)+\beta_{\epsilon \lambda}\right)$. Let $\left(\lambda_{k}\right)_{k \in \mathbb{N}}$ be a sequence in $] 0, \infty[$ with $\lim _{k \rightarrow \infty} \lambda_{k}=0$. Then there exists a $\left.K \in\right] 0, \infty[$ such that for all $k \in \mathbb{N}$ :

$(1 / \epsilon)\left\|A_{\epsilon}\left(\lambda_{k}\right)\right\|_{\left(H^{1}(\Omega)\right)^{\prime}},(1 / \epsilon)\left\|B_{\epsilon}\left(\lambda_{k}\right)\right\|_{\left(H^{1}(\Omega)\right)^{\prime}} \leq K$. Hence for all $k \in \mathbb{N}$ :

$\left\|\sqrt{n_{\epsilon, \lambda_{k}}}\right\|_{H^{1}(\Omega)},\left\|\sqrt{p_{\epsilon, \lambda_{k}}}\right\|_{H^{1}(\Omega)} \leq K+\sqrt{N}+\sqrt{P}$. Passing if necessary to a subsequence which is again denoted by $\left(\lambda_{k}\right)_{k \in \mathbb{N}}$ - we get

$$
\begin{gathered}
\sqrt{n_{\epsilon, \lambda_{k}}} \rightarrow \sqrt{n^{*}}, \sqrt{p_{\epsilon, \lambda_{k}}} \rightarrow \sqrt{p^{*}} \text { weakly in } H^{1}(\Omega) \text { as } k \rightarrow \infty, \\
n_{\epsilon, \lambda_{k}} \rightarrow n^{*}, p_{\epsilon, \lambda_{k}} \rightarrow p^{*} \text { strongly in } L^{2}(\Omega) \text { as } k \rightarrow \infty .
\end{gathered}
$$

We deduce from Theorem 1 that $n^{*}-p^{*}-C=0$. Due to the assumptions on $G_{1,2}$ we get by weak lower semi-continuity $\mathcal{E}_{i}\left(n^{*}, p^{*}\right)<\infty$. Hence $C$ is a $\left(G_{1}, G_{2}\right)$-quantum doping profile. 
Proof of Theorem 3:

a) By assumption we have $\mathcal{F}_{\epsilon}(n, p) \not \equiv \infty$ and $\mathcal{F}_{\epsilon} \geq 0$ on $\mathcal{C}_{0}$. Let $\left(n_{k}, p_{k}\right)$ be a minimizing sequence in $\mathcal{C}_{\circ}$ of $\mathcal{F}_{\epsilon}$. The $H^{1}(\Omega)$-norms of $\sqrt{n_{k}}, \sqrt{p_{k}}$ are uniformly bounded as $k \rightarrow \infty$. Hence there exists a $\left(n_{\epsilon \circ}, p_{\epsilon \circ}\right) \in L^{3}(\Omega)$ such that (possibly after passing to a subsequence)

$$
\sqrt{n_{k}} \rightarrow \sqrt{n_{\epsilon \circ}}, \sqrt{p_{k}} \rightarrow \sqrt{p_{\epsilon \circ}} \text { weakly in } H^{1}(\Omega) \text { as } k \rightarrow \infty .
$$

We easily check $\left(n_{\epsilon \circ}, p_{\epsilon \circ}\right) \in \mathcal{C}_{\circ}$. By weak lower sequential semi-continuity of $\mathcal{F}_{\epsilon}$, the pair $\left(n_{\epsilon \circ}, p_{\epsilon \circ}\right)$ is actually a minimizer of $\mathcal{F}_{\epsilon}$ in $\mathcal{C}_{\circ}$. The uniqueness of this minimizer follows from the strict convexity of $\mathcal{F}_{\epsilon}$.

b),c),d) For all $\lambda \in] 0, \infty[$ we have the inequalities $\mathcal{F}_{\epsilon}\left(n_{\epsilon \lambda}, p_{\epsilon \lambda}\right) \leq \mathcal{E}_{\epsilon \lambda}\left(n_{\epsilon \lambda}, p_{\epsilon \lambda}\right) \leq \mathcal{E}_{\epsilon \lambda}\left(n_{\epsilon \circ}, p_{\epsilon \circ}\right)=\mathcal{F}_{\epsilon}\left(n_{\epsilon \circ}, p_{\epsilon \circ}\right)$. Hence the $H^{1}(\Omega)$-norms of $\sqrt{n_{\epsilon \lambda}}, \sqrt{p_{\epsilon \lambda}}$ are uniformly bounded as $\lambda \rightarrow 0$. We conclude that there exists $\left(n^{*}, p^{*}\right) \in \mathcal{C}_{\circ}$ such that

$$
\sqrt{n_{\epsilon \lambda}} \rightarrow \sqrt{n^{*}}, \sqrt{p_{\epsilon \lambda}} \rightarrow \sqrt{p^{*}} \text { weakly in } H^{1}(\Omega) \text { as } \lambda \rightarrow 0 .
$$

By weak lower sequential continuity of $\mathcal{F}_{\epsilon}$, Theorem 1 and the previous estimate we get

$$
\lim _{\lambda \rightarrow 0} \mathcal{E}_{\epsilon \lambda}\left(n_{\epsilon \lambda}, p_{\epsilon \lambda}\right)=\mathcal{F}_{\epsilon}\left(n_{\epsilon \circ}, p_{\epsilon \circ}\right) .
$$

Due to the weak lower sequential continuity of $\mathcal{E}_{q u}$ and $\mathcal{E}_{i}$ we get from (3):

$$
\lim _{\lambda \rightarrow 0} \mathcal{E}_{q u}\left(n_{\epsilon \lambda}, p_{\epsilon \lambda}\right)=\mathcal{E}_{q u}\left(n_{\epsilon \circ}, p_{\epsilon \circ}\right) \quad, \quad \lim _{\lambda \rightarrow 0} \mathcal{E}_{i}\left(n_{\epsilon \lambda}, p_{\epsilon \lambda}\right)=\mathcal{E}_{i}\left(n_{\epsilon \circ}, p_{\epsilon \circ}\right) .
$$

From these identities and (3) we get:

i) $\left(n^{*}, p^{*}\right)$ is a minimizer of $\mathcal{F}_{\epsilon}$ in $\mathcal{C}_{\circ}$ and therefore $\left(n^{*}, p^{*}\right)=\left(n_{\epsilon \circ}, p_{\epsilon \circ}\right)$.

ii) $\lim _{\lambda \rightarrow 0} \lambda^{-2} \mathcal{E}_{e l}\left(n_{\epsilon \lambda}, p_{\epsilon \lambda}\right)=0$, which settles $\lim _{\lambda \rightarrow 0}\left\|\Phi_{\epsilon \lambda} / \lambda\right\|_{H^{1}(\Omega)}=0$.

iii) $\lim _{\lambda \rightarrow 0}\left\|\sqrt{n_{\epsilon \lambda}}\right\|_{H^{1}(\Omega)}=\left\|\sqrt{n_{\epsilon \circ}}\right\|_{H^{1}(\Omega)}, \lim _{\lambda \rightarrow 0}\left\|\sqrt{p_{\epsilon \lambda}}\right\|_{H^{1}(\Omega)}=\left\|\sqrt{p_{\epsilon \circ}}\right\|_{H^{1}(\Omega)}$. Hence by the uniform convexity of $H^{1}(\Omega)$ :

$$
\sqrt{n_{\epsilon \lambda}} \rightarrow \sqrt{n_{\epsilon \circ}}, \sqrt{p_{\epsilon \lambda}} \rightarrow \sqrt{p_{\epsilon \circ}} \text { strongly in } H^{1}(\Omega) \text { as } \lambda \rightarrow 0 .
$$

iv) We have

$$
\int_{\Omega} G_{1}\left(n_{\epsilon \lambda}\right) \rightarrow \int_{\Omega} G_{1}\left(n_{\epsilon \circ}\right), \int_{\Omega} G_{2}\left(p_{\epsilon \lambda}\right) \rightarrow \int_{\Omega} G_{2}\left(p_{\epsilon \circ}\right) \text { as } \lambda \rightarrow 0,
$$

as well as due to the continuity of $G_{1,2}$

$$
G_{1}\left(n_{\epsilon \lambda}\right) \rightarrow G_{1}\left(n_{\epsilon \circ}\right), G_{2}\left(p_{\epsilon \lambda}\right) \rightarrow G_{2}\left(p_{\epsilon \circ}\right) \mu \text {-a.e. as } \lambda \rightarrow 0 .
$$

As $0 \leq E_{\epsilon}<\infty$ we have - maybe after passing to a subnet -

$$
\sqrt{G_{1}\left(n_{\epsilon \lambda}\right)} \rightarrow \sqrt{G_{1}\left(n_{\epsilon \circ}\right)}, \sqrt{G_{2}\left(p_{\epsilon \lambda}\right)} \rightarrow \sqrt{G_{2}\left(p_{\epsilon \circ}\right)} \text { weakly in } L^{2}(\Omega) \text { as } \lambda \rightarrow 0,
$$

while (4) gives $\left\|\sqrt{G_{1}\left(n_{\epsilon \lambda}\right)}\right\|_{2} \rightarrow\left\|\sqrt{G_{1}\left(n_{\epsilon \circ}\right)}\right\|_{2},\left\|\sqrt{G_{2}\left(p_{\epsilon \lambda}\right)}\right\|_{2} \rightarrow\left\|\sqrt{G_{2}\left(p_{\epsilon \circ}\right)}\right\|_{2}$ as $\lambda \rightarrow 0$. Due to the uniform convexity of $L^{2}(\Omega)$ we get c). 
Proof of Lemma 5:

If $\limsup _{\lambda \rightarrow 0}\left\|n_{\epsilon \lambda}\right\|_{\infty}<\infty$ or $\limsup _{\lambda \rightarrow 0}\left\|p_{\epsilon \lambda}\right\|_{\infty}<\infty$, then by weak ${ }^{*}$-convergence in $L^{\infty}(\Omega)$ and Theorem $3, n_{\epsilon \circ} \in L^{\infty}(\Omega)$ or $p_{\epsilon \circ} \in L^{\infty}(\Omega)$. Due to $n_{\epsilon \circ}-p_{\epsilon \circ}=C \in L^{\infty}(\Omega)$ we get $n_{\epsilon \circ}, p_{\epsilon \circ} \in L^{\infty}(\Omega)$. Hence $\sqrt{n_{\epsilon \circ}}, \sqrt{p_{\epsilon \circ}} \in H^{1}(\Omega) \cap L^{\infty}(\Omega)$ gives $n_{\epsilon \circ}, p_{\epsilon \circ} \in H^{1}(\Omega)$ and therefore $C=n_{\epsilon \circ}-p_{\epsilon \circ} \in H^{1}(\Omega)$. Contradiction.

\section{Proof of Theorem 4:}

1.Step: We wish to prove that there exists an $\eta \in] 0,1[$ such that for all sufficiently small $\left.\lambda: \int_{\Omega} \sqrt{n_{\epsilon \lambda} p_{\epsilon \lambda}} \in\right] \eta, 1 / \eta\left[\right.$. To prove this statement we observe that by strong $L^{2}(\Omega)$ convergence of $\left(n_{\epsilon \lambda}, p_{\epsilon \lambda}\right)$ to $\left(n_{\epsilon \circ}, p_{\epsilon \circ}\right)$ (Theorem 3$)$ we have

$$
0 \leq \lim _{\lambda \rightarrow 0} \int_{\Omega} \sqrt{n_{\epsilon} p_{\epsilon \lambda}}=\int_{\Omega} \sqrt{n_{\epsilon \circ} p_{\epsilon \circ}}<\infty .
$$

To get a lower bound for $\int_{\Omega} \sqrt{n_{\epsilon \lambda} p_{\epsilon \lambda}}$ and sufficiently small $\lambda$, it suffices to prove that $\int_{\Omega} \sqrt{n_{\epsilon \circ} p_{\epsilon \circ}}>0$. This is shown in an indirect way. If $\int_{\Omega} \sqrt{n_{\epsilon \circ} p_{\epsilon \circ}}=0$, then it follows from $n_{\epsilon \circ}-p_{\epsilon \circ}=C$ that $n_{\epsilon \circ} \leq C^{+}, p_{\epsilon \circ} \leq C^{-}$, and therefore it follows from (A)d) that $N=\int_{\Omega} n_{\epsilon \circ} \leq \int_{\Omega} C^{+}<N, P=\int_{\Omega} p_{\epsilon \circ} \leq \int_{\Omega} C^{-}<P$. Contradiction.

2.Step: Due to Theorem 3 we have $\sqrt{n_{\epsilon \lambda}} \rightarrow \sqrt{n_{\epsilon \circ}}, \sqrt{p_{\epsilon \lambda}} \rightarrow \sqrt{p_{\epsilon \circ}}$ strongly in $H^{1}(\Omega)$ as $\lambda \rightarrow 0$. Due to assumptions (B) we have - maybe after passing to a subnet -

$$
\sqrt{n_{\epsilon \lambda}} g_{1}\left(n_{\epsilon \lambda}\right) \rightarrow \sqrt{n_{\epsilon \circ}} g_{1}\left(n_{\epsilon \circ}\right), \sqrt{p_{\epsilon \lambda}} g_{2}\left(p_{\epsilon \lambda}\right) \rightarrow \sqrt{p_{\epsilon \circ}} g_{2}\left(p_{\epsilon \circ}\right)
$$

weakly in $L^{6 / 5}(\Omega)$ as $\lambda \rightarrow 0$. Let $\phi \in W^{1, \infty}(\Omega)$. Testing the first two equations of (1) with $\phi \sqrt{p_{\epsilon \lambda}}, \phi \sqrt{n_{\epsilon \lambda}}$, respectively and subsequent addition gives

$$
\begin{aligned}
& -\epsilon^{2} \int_{\Omega} \nabla \sqrt{n_{\epsilon \lambda} p_{\epsilon \lambda}} \cdot \nabla \phi-2 \epsilon^{2} \int_{\Omega}\left(\nabla \sqrt{n_{\epsilon \lambda}} \cdot \nabla \sqrt{p_{\epsilon \lambda}}\right) \phi \\
= & \int_{\Omega} \sqrt{n_{\epsilon \lambda} p_{\epsilon \lambda}}\left(g_{1}\left(n_{\epsilon \lambda}\right)+g_{2}\left(p_{\epsilon \lambda}\right)+\alpha_{\epsilon \lambda}+\beta_{\epsilon \lambda}\right) \phi .
\end{aligned}
$$

On the other hand,

$$
\begin{aligned}
\int_{\Omega} \nabla \sqrt{n_{\epsilon \lambda} p_{\epsilon \lambda}} \cdot \nabla \phi & \rightarrow \int_{\Omega} \nabla \sqrt{n_{\epsilon \circ} p_{\epsilon \circ}} \cdot \nabla \phi \\
\int_{\Omega}\left(\nabla \sqrt{n_{\epsilon \lambda}} \cdot \sqrt{\nabla p_{\epsilon \lambda}}\right) \phi & \rightarrow \int_{\Omega}\left(\nabla \sqrt{n_{\epsilon \circ}} \cdot \nabla \sqrt{p_{\epsilon \circ}}\right) \phi \\
\int_{\Omega} \sqrt{n_{\epsilon \lambda} p_{\epsilon \lambda}}\left(g_{1}\left(n_{\epsilon \lambda}\right)+g_{2}\left(p_{\epsilon \lambda}\right)\right) \phi & \rightarrow \int_{\Omega} \sqrt{n_{\epsilon \circ} p_{\epsilon \circ}}\left(g_{1}\left(n_{\epsilon \circ}\right)+g_{2}\left(p_{\epsilon \circ}\right)\right) \phi
\end{aligned}
$$

as $\lambda \rightarrow 0$. Choosing $\phi=1$ we have for all $\lambda>0$

$$
\left(\alpha_{\epsilon \lambda}+\beta_{\epsilon \lambda}\right) \int_{\Omega} \sqrt{n_{\epsilon \lambda} p_{\epsilon \lambda}}=-2 \epsilon^{2} \int_{\Omega}\left(\nabla n_{\epsilon \lambda} \cdot \nabla p_{\epsilon \lambda}\right)-\int_{\Omega} \sqrt{n_{\epsilon \lambda} p_{\epsilon \lambda}}\left(g_{1}\left(n_{\epsilon \lambda}\right)+g_{2}\left(p_{\epsilon \lambda}\right)\right) .
$$

As the right-hand side of this equation is uniformly bounded as $\lambda \rightarrow 0$ and since there exists an $\eta \in] 0,1\left[\right.$ such that for all sufficiently small $\lambda>0$ the estimate $\int_{\Omega} \sqrt{n_{\epsilon \lambda} p_{\epsilon \lambda}} \in$ ]$\eta, 1 / \eta$ [ holds (see 1.Step), we get - maybe after passing to a subnet -

$$
\lim _{\lambda \rightarrow 0}\left(\alpha_{\epsilon \lambda}+\beta_{\epsilon \lambda}\right)=\gamma_{\epsilon} \in \mathbb{R}
$$

The proof of the remaining propositions can be left to the reader now. 
Proof of Lemma 6:

Certainly, for all $\epsilon>0$ :

$$
\inf _{\mathcal{C}_{\circ}} \mathcal{E}_{i} \leq \mathcal{E}_{i}\left(n_{\epsilon \circ}, p_{\epsilon \circ}\right) \leq \mathcal{F}_{\epsilon}\left(n_{\epsilon \circ}, p_{\epsilon, 0}\right)=E_{\epsilon}
$$

hence $\liminf \operatorname{in}_{\epsilon \rightarrow 0} E_{\epsilon} \geq \inf _{\mathcal{C}_{\circ}} \mathcal{E}_{i}$. On the other hand, for all $(n, p) \in \mathcal{C}_{\circ}$ :

$$
E_{\epsilon}=\mathcal{F}_{\epsilon}\left(n_{\epsilon \circ}, p_{\epsilon \circ}\right) \leq \mathcal{F}_{\epsilon}(n, p)=\epsilon^{2} \mathcal{E}_{q u}(n, p)+\mathcal{E}_{i}(n, p),
$$

and therefore $\lim \sup _{\epsilon \rightarrow 0} E_{\epsilon} \leq \inf _{\mathcal{C}_{\circ}} \mathcal{E}_{i}$.

\section{Proof of of Lemma 7:}

Due to [10] it is sufficient to prove: There exists a subsequence $\left(f_{n^{\prime}}\right)_{n^{\prime} \in \mathbb{N}^{\prime}}$ of $\left(f_{n}\right)_{n \in \mathbb{N}}$ such that

i) There exists a $\left.K_{1} \in\right] 0, \infty\left[\right.$ such that for all $n^{\prime} \in \mathbb{N}^{\prime}:\left\|f_{n^{\prime}}\right\|_{1} \leq K_{1}$.

ii) For all $B \in \sigma: \lim _{n^{\prime} \rightarrow \infty} \int_{B} f_{n^{\prime}} \in \mathbb{R}$.

Let $m=\min _{t \in \mathbb{R}} G(t)$.

For $L \in \mathbb{I N}$ let $\left.M_{L} \in\right] 0, \infty\left[\right.$ such that $\forall t \geq M_{L}: G(t) \geq L t$.

i) We have $\int_{S}\left|f_{n}\right|=\int_{\left\{\left|f_{n}\right|<M_{1}\right\}}\left|f_{n}\right|+\int_{\left\{\left|f_{n}\right| \geq M_{1}\right\}}\left|f_{n}\right| \leq \int_{\left\{\left|f_{n}\right|<M_{1}\right\}} M_{1}+\int_{\left\{\left|f_{n}\right| \geq M_{1}\right\}} G\left(\left|f_{n}\right|\right)$ $\leq M_{1} \mu(S)+K \equiv K_{1}$.

ii) We have for all $L \in \mathbb{I N}$ : The sequence $\left(f_{n} 1_{\left\{\left|f_{n}\right| \leq M_{L}\right\}}\right)_{n \in \mathbb{N}}$ is uniformly bounded in $L^{\infty}(S, \sigma, \mu)=\left(L^{1}(S, \sigma, \mu)\right)^{\prime}$. As $L^{1}(S, \sigma, \mu)$ is separable, the unit sphere in $L^{\infty}(S, \sigma, \mu)$ is $L^{\infty}(S, \sigma, \mu)$-weak* sequentially compact. By a diagonalization procedure one can therefore extract a subsequence $\left(f_{n^{\prime}}\right)_{n^{\prime} \in \mathbb{N}^{\prime}}$ such that for all $L \in \mathbb{N}$ : There exists a $h_{L} \in L^{\infty}(S, \sigma, \mu)$ such that

$$
f_{n^{\prime}} 1_{\left\{\left|f_{n}\right|<M_{L}\right\}} \rightarrow h_{L} \text { weak }^{*} \text { in } L^{\infty}(S, \sigma, \mu) .
$$

Let $B \in \sigma$. We shall prove: $\left(\int_{B} f_{n^{\prime}}\right)_{n^{\prime} \in \mathbb{N}^{\prime}}$ is a Cauchy sequence in $\mathbb{R}$. Let $\delta>0$. Take $L_{0}=L_{0}(\delta) \in \mathbb{I N}$ such that $4 \sup _{n \in \mathbb{N}} \int_{S} G\left(\left|f_{n}\right|\right) \leq L_{0} \delta$. Then for all $n^{\prime} \in \mathbb{I N}^{\prime}$ :

$$
\left|\int_{B} f_{n^{\prime}} 1_{\left\{\left|f_{n^{\prime}}\right| \geq M_{L_{0}}\right\}}\right| \leq \int_{\left\{\left|f_{n^{\prime}}\right| \geq M_{L_{0}}\right\}}\left|f_{n^{\prime}}\right| \leq \delta / 4 \text {. }
$$

As $\mu(S)<\infty$ we have $1_{B} \in L^{1}(S, \sigma, \mu)$ and therefore there exists by weak* convergence of $\left(f_{n^{\prime}} 1_{\left\{\left|f_{n^{\prime}}\right| \leq M_{\left.L_{0}\right\}}\right\}}\right)_{n^{\prime} \in \mathbb{N}^{\prime}}$ in $L^{\infty}(S, \sigma, \mu)$ an $n * \in \mathbb{N}^{\prime}$ such that for all $n^{\prime}, m^{\prime} \in \mathbb{N}^{\prime}$ : If $n * \leq n^{\prime}, m^{\prime}$, then

$$
\left|\int_{B} f_{n^{\prime}} 1_{\left\{\left|f_{n^{\prime}}\right|<M_{L_{0}}\right\}}-\int_{B} f_{m^{\prime}} 1_{\left\{\left|f_{m^{\prime}}\right|<M_{L_{0}}\right\}}\right| \leq \delta / 4 \text {. }
$$

Combining equations $(5),(6)$ we conclude that $\left(\int_{B} f_{n^{\prime}}\right)_{n^{\prime} \in \mathbb{N}^{\prime}}$ is actually a Cauchy sequence in $\mathbb{R}$.

Proof of Theorem 5:

Let $\left(\epsilon_{k}\right)_{k \in \mathbb{N}}$ be a sequence in $] 0, \infty\left[\right.$ with $\lim _{k \rightarrow \infty} \epsilon_{k}=0$. To simplify notations let $n_{k}=n_{\epsilon_{k} \circ}, p_{k}=p_{\epsilon_{k}}$. To prove A1. it is sufficient to show that there exists a subsequence $\left(\epsilon_{k^{\prime}}\right)_{k^{\prime} \in \mathbb{N}^{\prime}}$ such that

$$
n_{k^{\prime}} \rightarrow n_{\circ \circ}, p_{k^{\prime}} \rightarrow p_{\circ \circ} \text { strongly in } L^{1}(\Omega) \text { as } k^{\prime} \rightarrow \infty .
$$


As $\lim _{\epsilon \rightarrow 0} E_{\epsilon}=\inf _{\mathcal{D}} \mathcal{E}_{i}$ there is $\left.K \in\right] 0, \infty\left[\right.$ such that for all $k \in \mathbb{I N}: \mathcal{E}_{i}\left(n_{k}, p_{k}\right)=$ $\int_{\Omega} G_{1}\left(n_{k}\right)+\int_{\Omega} G_{2}\left(p_{k}\right) \leq K$. It follows from Lemma 7 that there exists subsequences $\left(n_{k^{\prime}}\right)_{k^{\prime} \in \mathbb{N}^{\prime}},\left(p_{k^{\prime}}\right)_{k^{\prime} \in \mathbb{N}^{\prime}}$ and $n *, p * \in L^{1}(\Omega)$ such that

$$
n_{k^{\prime}} \rightarrow n *, p_{k^{\prime}} \rightarrow p * \text { weakly in } L^{1}(\Omega) \text { as } k^{\prime} \rightarrow \infty .
$$

Clearly, $(n *, p *) \in \mathcal{D}$. By lower sequential continuity of $\mathcal{E}_{i}$ we have

$$
\mathcal{E}_{i}(n *, p *) \leq \lim \inf _{k^{\prime} \rightarrow \infty} \mathcal{E}_{i}\left(n_{k^{\prime}}, p_{k^{\prime}}\right)=\inf _{\mathcal{D}} \mathcal{E}_{i}
$$

As $\left(n_{\circ \circ}, p_{\circ \circ}\right)$ is the unique minimizer of $\mathcal{E}_{i}$ in $\mathcal{D}$ we have $n *=n_{\circ \circ}, p *=p_{\circ \circ}$. It follows from the lower sequential continuity of $\mathcal{E}_{i}$ and from $\mathcal{E}_{i}\left(n_{\circ \circ}, p_{\circ \circ}\right)=\lim _{k^{\prime} \rightarrow \infty} \mathcal{E}_{i}\left(n_{k^{\prime}}, p_{k^{\prime}}\right)$ that

$$
\lim _{k^{\prime} \rightarrow \infty} \int_{\Omega} G_{1}\left(n_{k^{\prime}}\right)=\int_{\Omega} G_{1}\left(n_{\circ \circ}\right), \lim _{k^{\prime} \rightarrow \infty} \int_{\Omega} G_{2}\left(p_{k^{\prime}}\right)=\int_{\Omega} G_{2}\left(p_{\circ \circ}\right) .
$$

Hence we get from the compactness-by-convexity principle (Lemma 8)

$$
n_{k^{\prime}} \rightarrow n_{\circ \circ}, p_{k^{\prime}} \rightarrow p_{\circ \circ} \text { strongly in } L^{1}(\Omega) \text { as } k^{\prime} \rightarrow \infty \text {. }
$$

This settles A1. Proposition A2. follows immediately from $\lim _{\epsilon \rightarrow 0} \mathcal{E}_{q u}\left(n_{\epsilon \circ}, p_{\epsilon \circ}\right)=0$. To prove A3. assume that $\left(n_{\circ \circ}, p_{\circ \circ}\right) \in \mathcal{C}_{\circ}$. Then for all $k^{\prime} \in \mathbb{N}^{\prime}$ :

$$
\begin{aligned}
& \epsilon_{k^{\prime}}^{2} \int_{\Omega}\left|\nabla \sqrt{n_{k^{\prime}}}\right|^{2}+\epsilon_{k^{\prime}}^{2} \int_{\Omega}\left|\nabla \sqrt{p_{k^{\prime}}}\right|^{2}+\mathcal{E}_{i}\left(n_{k^{\prime}}, p_{k^{\prime}}\right) \\
& \quad \leq \epsilon_{k^{\prime}}^{2} \int_{\Omega}\left|\nabla \sqrt{n_{\circ \circ}}\right|^{2}+\epsilon_{k^{\prime}}^{2} \int_{\Omega}\left|\nabla \sqrt{p_{\circ \circ}}\right|^{2}+\mathcal{E}_{i}\left(n_{\circ \circ}, p_{\circ \circ}\right) .
\end{aligned}
$$

As $\left(n_{\circ \circ}, p_{\circ \circ}\right)$ is the minimizer of $\mathcal{E}_{i}$ in $\mathcal{D}$ we get from this estimate $\int_{\Omega}\left|\nabla \sqrt{n_{k^{\prime}}}\right|^{2}+$ $\int_{\Omega}\left|\nabla \sqrt{p_{k^{\prime}}}\right|^{2} \leq \int_{\Omega}\left|\nabla \sqrt{n_{\circ \circ}}\right|^{2}+\int_{\Omega}\left|\nabla \sqrt{p_{\circ \circ}}\right|^{2}$, where the right-hand side is bounded independently of $k^{\prime} \in \mathbb{I}^{\prime}$. By passing to a subsequence $\left(n_{k^{\prime \prime}}, p_{k^{\prime \prime}}\right)_{k^{\prime \prime} \in \mathbb{N}^{\prime \prime}}$ we have weak $H^{1}(\Omega)$-convergence of $\left(n_{k^{\prime \prime}}, p_{k^{\prime \prime}}\right)$ to $\left(n_{\circ \circ}, p_{\circ \circ}\right)$ and we deduce from lower sequential continuity

$$
\int_{\Omega}\left|\nabla \sqrt{n_{\circ \circ}}\right|^{2}+\int_{\Omega}\left|\nabla \sqrt{p_{\circ}}\right|^{2} \leq \lim \inf _{k^{\prime \prime} \rightarrow \infty}\left(\int_{\Omega}\left|\nabla \sqrt{n_{k^{\prime \prime}}}\right|^{2}+\int_{\Omega}\left|\nabla \sqrt{p_{k^{\prime}}}\right|^{2}\right)
$$

hence

$$
\int_{\Omega}\left|\nabla \sqrt{n_{\circ \circ}}\right|^{2}=\lim _{k^{\prime \prime} \rightarrow \infty} \int_{\Omega}\left|\nabla \sqrt{n_{k^{\prime \prime}}}\right|^{2}, \int_{\Omega}\left|\nabla \sqrt{p_{\circ \circ}}\right|^{2}=\lim _{k^{\prime \prime} \rightarrow \infty} \int_{\Omega}\left|\nabla \sqrt{p_{k^{\prime \prime}}}\right|^{2}
$$

and the result follows from the uniform convexity of $H^{1}(\Omega)$.

\section{References}

[1] Adams, R. 1975 Sobolev Spaces. New York. Academic Press.

[2] Markowich, P. A. 1986 The Stationary Semiconductor Device Equations. New York. Springer.

[3] Markowich, P. A. \& Ringhofer, C. A. \& Schmeiser, C. 1990 Semiconductor Equations. New York. Springer. 
[4] Markowich, P. A. \& Unterreiter, A. 1993 Vacuum Solutions of the Stationary Drift-Diffusion Model. Annali della Scuola Normale Superiore di Pisa, XX, 371-386.

[5] Pinnau, R. \& Unterreiter, A. 1999 The Stationary Current-Voltage Characteristics of the Quantum Drift Diffusion Model. to appear in SIAM Journal on Numerical Analysis.

[6] Rudin, W. 1987 Real and Complex Analysis. (3rd edn). New York. McGraw-Hill.

[7] Struwe, M. 1990 Variational Methods. Berlin. Springer.

[8] Unterreiter, A. 1995 Vacuum and Non-Vacuum Solutions of the Quasi Hydrodynamic Semiconductor Model in Thermal Equilibrium. Mathematical Methods in the Applied Sciences 18, 225-254.

[9] Unterreiter, A. 1997 The Thermal Equilibrium Solution of a Generic Bipolar Quantum Hydrodynamic Model. Communications in Mathematical Physics 188, 6988.

[10] YosidA, K. 1995 Functional Analysis. (6th edn). Berlin. Springer.

[11] Ziemer, W. P. 1989 Weakly Differentiable Functions. New York. Springer. 